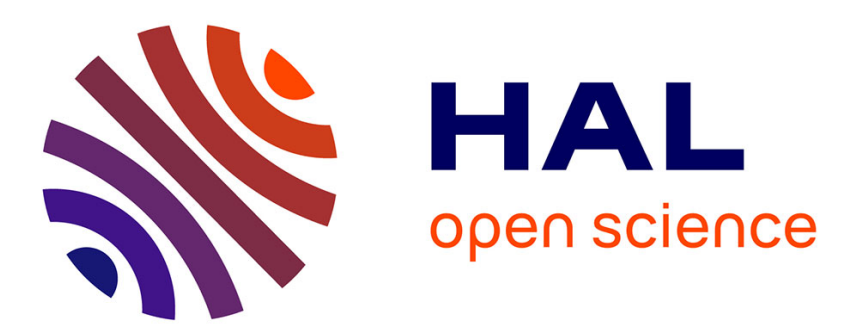

\title{
A Hamiltonian system for interacting Benjamin-Feir resonances
}

\author{
Tounsia Benzekri, Cristel Chandre, Ricardo Lima, Michel Vittot
}

\section{To cite this version:}

Tounsia Benzekri, Cristel Chandre, Ricardo Lima, Michel Vittot. A Hamiltonian system for interacting Benjamin-Feir resonances. Journal of Physics A: Mathematical and Theoretical, 2005, 38, pp.5381. 10.1088/0305-4470/38/24/001 . hal-00002312

\section{HAL Id: hal-00002312 https://hal.science/hal-00002312}

Submitted on 26 Jul 2004

HAL is a multi-disciplinary open access archive for the deposit and dissemination of scientific research documents, whether they are published or not. The documents may come from teaching and research institutions in France or abroad, or from public or private research centers.
L'archive ouverte pluridisciplinaire HAL, est destinée au dépôt et à la diffusion de documents scientifiques de niveau recherche, publiés ou non, émanant des établissements d'enseignement et de recherche français ou étrangers, des laboratoires publics ou privés. 


\title{
A Hamiltonian system for interacting Benjamin-Feir resonances
}

\author{
T. Benzekri *, C. Chandre, R. Lima and M. Vittot \\ Centre de Physique Théorique, CNRS UMR 6207, \\ Luminy - case 907, F-13288 Marseille cedex 9, France
}

\begin{abstract}
In this paper, we present a model describing the time evolution of two dimensional surface waves in gravity and infinite depth. The model of six interacting modes derives from the normal form of the system describing the dynamics of surface waves and is governed by a Hamiltonian system of equations of cubic order in the amplitudes of the waves. We derive a Hamiltonian system with two degrees of freedom from this Hamiltonian using conserved quantities. The interactions are those of two coupled Benjamin-Feir resonances. The temporal evolution of the amplitude of the different modes is described according to the parameters of the system. In particular, we study the energy exchange produced by the modulations of the amplitudes of the modes. The evolution of the modes reveals a chaotic dynamics.
\end{abstract}

PACS numbers: $05.45 .-\mathrm{a} ; 47.35 .+\mathrm{i} ; 47.52 .+\mathrm{j}$

\footnotetext{
* Corresponding Author: tel (+33) 491269522, fax (+33) 491269553
} 


\section{INTRODUCTION}

In 1967, Benjamin and Feir [1] studied the linear stability of periodic solutions of permanent form for gravity surface waves in infinite depth. They showed that wave trains are unstable to modulation perturbation i.e that instabilities occur due to the interaction of the carrier wave and two wave perturbations. This kind of instability has been first found by Lighthill [2] and confirmed theoretically and experimentally by Benjamin and Feir. This result was also confirmed by Zakharov [3] using a Hamiltonian formalism for the surface wave problem. This instability was interpreted in terms of resonance conditions between the carrier wave and the two side bands. Later in 1977, Lake et al. [4] observed in wave tank experiments the first modulation and demodulation of the recurrence Fermi-PastaUlam phenomenon [5].

Many works using the Hamiltonian formalism which was first derived by Zakharov, have been carried out to understand the time evolution of the resonant interaction phenomenon [6, 7, 8]. We refer in particular, for three dimensions, to Shemer and Stiassnie [9], Stiassnie and Shemer [10], Shrira et al. [11] and Badulin et al. [12]. In two dimensions, we refer to Benzekri et al. [13], Caponi et al. [14] who use a seven modes model in order to show a chaotic behavior. In order to study the long time evolution of surface waves, Zufiria [15] derived from the Zakharov water waves equations a simple model of three interacting modes [Eq.(10) in Ref. [15]] which leads to a chaotic behavior. However this model is not invariant by time reversing symmetry.

Other works using the Hamiltonian formalism in fluid interfaces were carried out in Refs. 16, 17.

In order to give a qualitative description of the spatio-temporal evolution of the dynamical system described by a Hamiltonian water wave system, we propose in our work a simple model using all the symmetries of the initial equations of surface waves. In particular, we use the time reversing symmetry to derive a system of Hamilton's equations describing the dynamics of Benjamin-Feir resonant waves. This symmetry plays an important role in the study of the system. The evolution of the complex amplitudes of the modes is governed by a cubic dynamical system of equations. The system is then a set of six ordinary differential equations. We show the existence of invariants that reduces this system to two degrees of freedom. 
We use a geometrical approach to understand the energy exchange mechanism among the modes of the wave. For some initial conditions, we find that the Benjamin-Feir resonance leads to a continual periodic energy transfer from one component of the wave to the others. We also find by numerical simulations an irregular transfer of energy among the modes of the wave. The energy transfer of this kind is related to the existence of chaos in our system.

This paper is organized as follows: In Sec. III, we describe the model which is an approximation of the Zakharov Hamiltonian water waves system developed by Krazitskii [18]. In this model, the interactions require the coexistence of two resonating four-wave interactions. The Hamiltonian of this model written in normal form is of order four in the amplitudes of the wave.

The system has six degrees of freedom, then having six equations for the complex amplitudes. We find four integrals of motion thanks to the Manley-Rowe relations. Therefore, we reduce the model to a Hamiltonian system with two degrees of freedom.

The knowledge of these invariants allows us to subdivide the phase space in different regions and determine analytical solutions like traveling and standing waves. In particular, we analyze how the system behaves starting from different initial conditions. Section III] is devoted to the study of the dynamics of the system with only one resonance. The study of the chaotic dynamics with the two interacting resonances is done in Sec. एV.

\section{THE MODEL}

In an inviscid and incompressible fluid approximation, the equations of the twodimensional gravity waves on the surface of deep water read as follows [3]:

$$
\begin{aligned}
& \Delta \varphi(x, y, t)=0, \quad \text { for }-\infty<y<\eta(x, t), \\
& g \eta(x, t)+\frac{\partial \varphi}{\partial t}(x, y, t)+\frac{1}{2}|\nabla \varphi(x, y, t)|^{2}=0, \quad \text { for } y=\eta(x, t), \\
& \frac{\partial \eta}{\partial t}(x, y, t)+\frac{\partial \varphi}{\partial x}(x, y, t) \frac{\partial \eta}{\partial x}(x, y, t)-\frac{\partial \varphi}{\partial y}(x, y, t)=0, \quad \text { for } y=\eta(x, t), \\
& \lim _{y \rightarrow-\infty} \nabla \varphi(x, y, t)=0,
\end{aligned}
$$

where the unknown variables are the surface shape of the wave $\eta$, and the stream function $\varphi$ and $g$ is the strength of the gravity. Zakharov [3] showed that these equations can be 
expressed in a Hamiltonian form:

$$
\frac{d a_{k}}{d t}=-i \frac{\partial H}{\partial a_{k}^{*}}, \quad \text { for } k \in \mathbb{R}
$$

where the complex amplitudes $a_{k}$ are linear combinations of the Fourier coefficients of $\eta(x, t)$ and of the free surface velocity potential $\psi(x, t)$ defined by

$$
\psi(x, t)=\varphi(x, \eta(x, t), t) .
$$

If we restrict ourself to the spatially periodic waves then $k \in \mathbb{Z}$. More precisely, if we define the Fourier expansion of the wave

$$
\eta(x, t)=\frac{1}{2 \pi} \sum_{k=-\infty}^{+\infty} \eta_{k} e^{i k x},
$$

the relation between the coefficients $\eta_{k}$ and the complex symplectic coordinates $a_{k}$ is

$$
\eta_{k}(t)=\frac{g^{-1 / 4}}{\sqrt{2}}|k|^{1 / 4}\left(a_{k}+a_{-k}^{*}\right)
$$

The Hamiltonian is given by the total energy of the system when expressed as function of the $a_{k}$ (see Ref. [18]).

Our model is obtained by truncating the general (infinite dimensional) Hamiltonian derived by Zakharov in the form stated by Krazitszkii [18] up to order four and retaining a minimal set of modes in order to describe a Benjamin-Feir instability for gravity surface waves in infinite depth.

In order to preserve time reversal symmetry, this minimal number of complex modes is six, $\pm k_{1}, \pm k_{2}, \pm k_{3}$ that leads to a set of two coupled resonances verifying:

$$
k_{1}+k_{2}=2 k_{3}, \quad \text { and } \quad \omega_{k_{1}}+\omega_{k_{2}}=2 \omega_{k_{3}},
$$

together with the dispersion relation:

$$
\omega_{k}=\sqrt{g|k|}, \quad \text { for } \quad k= \pm k_{1}, \pm k_{2}, \pm k_{3}
$$

Dyachencho and Zakharov [19] described the general families of such type of resonances. In our case this leads to only one family of resonances, namely

$$
k_{1}=-a, \quad k_{2}=9 a, \quad k_{3}=4 a, \quad \text { for } a \in \mathbb{Z}^{*} .
$$


For the simplicity of the exposition, the modes will be denoted by $a_{j}$ instead of $a_{k_{j}}$ for $j=1,2,3$ and $a_{-j}$ instead of $a_{-k_{j}}$.

It is clear from Eq. (6) that such resonances are built from central carriers, $a_{3}$ and $a_{-3}$, and two equally distant side bands.

The expression of the Hamiltonian in the variables $a_{j}$ up to order 4 is given in the Appendix. Then following Ref. [18], we perform a canonical transformation from the variables $a_{j}$ to the variables $b_{j}$ such that the Hamiltonian is mapped into its normal form. We rederive it in the Appendix.

The Hamiltonian of the system becomes in the variables $b_{j}$

$$
\begin{aligned}
H= & \sum_{j=1,2,3} \omega_{j}\left(\left|b_{j}\right|^{2}+\left|b_{-j}\right|^{2}\right)+\sum_{j=1,2,3} U_{j}\left(\left|b_{j}\right|^{4}+\left|b_{-j}\right|^{4}-4\left|b_{j}\right|^{2}\left|b_{-j}\right|^{2}\right) \\
& +V_{21}\left(\left|b_{2}\right|^{2}\left|b_{1}\right|^{2}+\left|b_{-2}\right|^{2}\left|b_{-1}\right|^{2}\right)+V_{-21}\left(\left|b_{2}\right|^{2}\left|b_{-1}\right|^{2}+\left|b_{-2}\right|^{2}\left|b_{1}\right|^{2}\right) \\
& +V_{31}\left(-\left|b_{3}\right|^{2}\left|b_{1}\right|^{2}-\left|b_{-3}\right|^{2}\left|b_{-1}\right|^{2}\right)+V_{-31}\left(\left|b_{3}\right|^{2}\left|b_{-1}\right|^{2}+\left|b_{-3}\right|^{2}\left|b_{1}\right|^{2}\right) \\
& +V_{23}\left(\left|b_{2}\right|^{2}\left|b_{3}\right|^{2}+\left|b_{-2}\right|^{2}\left|b_{-3}\right|^{2}\right)+V_{-23}\left(-\left|b_{2}\right|^{2}\left|b_{-3}\right|^{2}-\left|b_{-2}\right|^{2}\left|b_{3}\right|^{2}\right) \\
& +\frac{1}{2} W\left(b_{1} b_{2} b_{3}^{* 2}+b_{1}^{*} b_{2}^{*} b_{3}^{2}+b_{-1} b_{-2} b_{-3}^{* 2}+b_{-1}^{*} b_{-2}^{*} b_{-3}^{2}\right),
\end{aligned}
$$

where the coefficients $U_{j}, V_{i j}$ and $W$ are positive. The values of all these coefficients are given in the Appendix .

The equations of motion are :

$$
\frac{d b_{j}}{d t}=-i \frac{\partial H}{\partial b_{j}^{*}}
$$

for $j=-1,-2,-3,1,2,3$.

We notice that, in the infinite dimensional case, as showed by Dyachenko and Zakharov [19] and Craig and Worfolk [20], the canonical transformation that eliminates the cubic terms also cancels the fourth order terms. In our case, starting from the finite dimensional truncation, the Benjamin-Feir fourth order terms are instead present in the normal form. This shows a difference between the infinite dimensional case and our finite dimensional model. A similar Hamiltonian model for fluid interfaces has been derived in Ref. [16] where Benjamin-Feir resonances appear even in the infinite dimensional case due to the presence of the non-vanishing density of the upper fluid.

Using some scaling relations, it is possible to assume that $\omega_{1}=1, \omega_{2}=3$ and $\omega_{3}=2$. Indeed, if we rescale the variables $b_{j}$ by a factor $\sqrt{\lambda}$ for $\lambda \in \mathbb{R}^{*+}$, i.e., if we replace the 
Hamiltonian $H\left(b_{j}, b_{j}^{*}\right)$ by $\lambda H\left(b_{j} / \sqrt{\lambda}, b_{j}^{*} / \sqrt{\lambda}\right)$ which is a transformation that preserves the equations of motion, and if we rescale time by the same factor $\lambda$, i.e., we multiply the Hamiltonian by $\lambda$, then it is easy to see that the quartic part of the Hamiltonian is unchanged and the quadratic part is multiplied by $\lambda$. By choosing $\lambda=1 / \sqrt{g|a|}$, it means that we can consider that the frequencies are $\omega_{1}=1, \omega_{2}=3$ and $\omega_{3}=2$ without loss of generality.

As we shall see, the existence of several integrals of the motion in our system, despite the fact that they are not sufficient to turn it into an integrable one, allows to reduce the number of degrees of freedom from six to two and to have important information about the dynamics in phase space.

The equations of evolution of $\left|b_{1}\right|^{2},\left|b_{2}\right|^{2}$ and $\left|b_{3}\right|^{2}$ are

$$
\begin{aligned}
& \frac{d}{d t}\left|b_{1}\right|^{2}=i \frac{W}{2}\left(b_{1} b_{2} b_{3}^{* 2}-b_{1}^{*} b_{2}^{*} b_{3}^{2}\right), \\
& \frac{d}{d t}\left|b_{2}\right|^{2}=i \frac{W}{2}\left(b_{1} b_{2} b_{3}^{* 2}-b_{1}^{*} b_{2}^{*} b_{3}^{2}\right), \\
& \frac{d}{d t}\left|b_{3}\right|^{2}=-i W\left(b_{1} b_{2} b_{3}^{* 2}-b_{1}^{*} b_{2}^{*} b_{3}^{2}\right) .
\end{aligned}
$$

Therefore we see that the following two quantities are conserved

$$
\begin{aligned}
& \mathbb{I}_{+}=2\left|b_{1}\right|^{2}+\left|b_{3}\right|^{2}, \\
& \mathbb{J}_{+}=\left|b_{1}\right|^{2}+3\left|b_{2}\right|^{2}+2\left|b_{3}\right|^{2} .
\end{aligned}
$$

In what follows, we will also use a linear combination of these two conserved quantities

$$
\mathbb{K}_{+}=\left|b_{1}\right|^{2}-\left|b_{2}\right|^{2}=\frac{1}{3}\left(2 \mathbb{I}_{+}-\mathbb{J}_{+}\right) .
$$

By defining $N_{j} \equiv\left|b_{j}\right|^{2}$ for $j=1,2,3$, the constants of motion become:

$$
\begin{aligned}
& \mathbb{I}_{+}=2 N_{1}+N_{3}, \\
& \mathbb{J}_{+}=N_{1}+3 N_{2}+2 N_{3} .
\end{aligned}
$$

Similarly, we have two additional conserved quantities for the other resonance

$$
\begin{aligned}
& \mathbb{I}_{-}=2 N_{-1}+N_{-3}, \\
& \mathbb{J}_{-}=N_{-1}+3 N_{-2}+2 N_{-3},
\end{aligned}
$$

where $N_{-j} \equiv\left|b_{-j}\right|^{2}$ for $j=1,2,3$.

It can be checked that the four integrals $\mathbb{I}_{+}, \mathbb{J}_{+}, \mathbb{I}_{-}, \mathbb{J}_{-}$are independent and in involution. 
The key point to be noticed here, is the fact that each one of these first integrals depends on the variables of one resonance alone even if, as it will be seen, there is a dynamical interaction of the two sets of variables.

Let us now look at the structure of the invariant surfaces in phase space in view of the previous integrals of motion. Even if they can not completely fix the orbits of the system, they help to understand many properties of the different dynamical regimes observed in this model.

In the space $\mathbb{E}_{+}=\left(N_{1}, N_{2}, N_{3}\right)$ (respectively $\left.\mathbb{E}_{-}=\left(N_{-1}, N_{-2}, N_{-3}\right)\right)$, $\mathbb{J}_{+}=$const. (resp. $\mathbb{J}_{-}=$const.) defines a portion of a plane, that we represent in Fig. 1. On the other hand, the intersection of this set with $\mathbb{I}_{+}=$const. (resp. $\mathbb{I}_{-}=$const.) defines a family of segments $C_{+}\left(\mathbb{I}_{+}\right)\left(\operatorname{resp} . C_{-}\left(\mathbb{I}_{-}\right)\right)$.

Let us consider the structure of $\mathbb{E}_{+}$and let us fix $\mathbb{J}_{+} \neq 0$. For $\mathbb{I}_{+}=0$, the set $C_{+}(0)$ reduces to one point $P$ whose coordinates are $P=\left(0, \mathbb{J}_{+} / 3,0\right)$.

For $0<\mathbb{I}_{+}<\frac{\mathbb{J}_{+}}{2}$, the intersection of the two invariant surfaces in $\mathbb{E}_{+}, C_{+}\left(\mathbb{I}_{+}\right)$is a segment close to $P$.

When $\mathbb{I}_{+}=\mathbb{J}_{+} / 2, C_{+}\left(\mathbb{J}_{+} / 2\right)$ is a segment with one extremity $T=\left(0,0, \mathbb{J}_{+} / 2\right)$. We notice that on this curve we have $N_{1}=N_{2}$.

For $\mathbb{J}_{+} / 2<\mathbb{I}_{+}<2 \mathbb{J}_{+}$, the set $C_{+}\left(\mathbb{I}_{+}\right)$is a segment close to $S=\left(\mathbb{I}_{+}, 0,0\right)$.

For $\mathbb{I}_{+}=2 \mathbb{J}_{+}$, the intersection is the point $S$.

For $\mathbb{I}_{+}>2 \mathbb{J}_{+}$or for $\mathbb{I}_{+}<0$ the intersection is empty.

We notice that $\mathbb{E}_{+} \times \mathbb{E}_{-}$needs to be supplemented by additional variables like the six angles $\Phi_{j}$ of the complex variables $b_{j}$ in order to build the phase space. Therefore, since the projection of an orbit on $\mathbb{E}_{+} \times \mathbb{E}_{-}$alone is only part of the information we must look at it as a partial, although useful, information on the dynamics. This is why, in each case we shall supplement its description with additional information in order to make a complete characterization of the dynamics. 


\section{DYNAMICS WITH ONE RESONANCE}

To get insight into the full dynamics, we begin with simple and integrable situations which will allow us to understand the general case with two resonances. We start exploring the phase space by choosing initial conditions leading to simple situations for which we can exhibit different kinds of shapes of the wave. A complete description of phase space is given by a numerical analysis.

It follows from the conserved quantities, that an orbit corresponding to an initial condition with all null coordinates in one of the two resonance variables $N_{j}$ will keep this property for any time. For instance, we assume that $\mathbb{J}_{-}=0$. This implies that $N_{-1}=N_{-2}=N_{-3}=0$ for all time. Thus, we first study the dynamics with only one resonance which is an integrable case since Hamiltonian (12) has three degrees of freedom and three conserved quantities (e.g. $\left.H, \mathbb{I}_{+}, \mathbb{K}_{+}\right)$. Hamiltonian (8) describing the dynamics in the variables $\left(b_{1}, b_{2}, b_{3}\right)$ becomes :

$$
\begin{aligned}
H= & \left|b_{1}\right|^{2}+3\left|b_{2}\right|^{2}+2\left|b_{3}\right|^{2}+U_{1}\left|b_{1}\right|^{4}+U_{2}\left|b_{2}\right|^{4}+U_{3}\left|b_{3}\right|^{4} \\
& +V_{21}\left|b_{2}\right|^{2}\left|b_{1}\right|^{2}-V_{31}\left|b_{3}\right|^{2}\left|b_{1}\right|^{2}+V_{23}\left|b_{2}\right|^{2}\left|b_{3}\right|^{2} \\
& +\frac{1}{2} W\left(b_{1} b_{2} b_{3}^{* 2}+b_{1}^{*} b_{2}^{*} b_{3}^{2}\right) .
\end{aligned}
$$

In what follows, we describe the dynamics on the sets $C_{+}\left(\mathbb{I}_{+}\right)$described in the previous section (see Fig. 1). We start with the simplest cases which are the three points $P, T$ and $S$ and the segment $(P S)$ (one of the boundaries of the triangle $\mathbb{E}_{+}$) for which we can exhibit analytical expressions for the shape of the wave $\eta(x, t)$.

\section{A. Dynamics of orbits of $P, T, S$}

First we consider the orbits corresponding to the point $P$ of $\mathbb{E}_{+}$(where $\mathbb{I}_{+}=0$ ). The equations of motion read

$$
\begin{aligned}
& N_{1} \equiv\left|b_{1}\right|^{2}=0, \\
& N_{3} \equiv\left|b_{3}\right|^{2}=0, \\
& N_{2} \equiv\left|b_{2}\right|^{2}=\frac{\mathbb{J}_{+}}{3},
\end{aligned}
$$




$$
i \frac{d b_{2}}{d t}=\left(3+2 U_{2}\left|b_{2}\right|^{2}\right) b_{2}
$$

The solution in the variables $b_{j}$ is then

$$
\begin{aligned}
& b_{2}(t)=\sqrt{\frac{\mathbb{J}_{+}}{3}} \exp \left(-i\left(3+2 U_{2} \frac{\mathbb{J}_{+}}{3}\right) t-i \Phi\right), \\
& b_{j}(t)=0, \quad \text { for } j=1,3 .
\end{aligned}
$$

Therefore for any $\mathbb{J}_{+}>0$ these orbits are periodic in time. Since we have only one resonance, the original variables $a_{j}$ are equal to $b_{j}$ (see Remark 1 of the Appendix).

Thus the expression of the shape of the wave is:

$$
\eta(x, t)=\frac{\sqrt{3} g^{-1 / 4}}{2 \pi \sqrt{2}}\left(b_{2}(t) \exp (9 i x)+b_{2}^{*}(t) \exp (-9 i x)\right)
$$

which is also

$$
\eta(x, t)=\eta_{0} \cos \left(9\left(x-c_{2} t\right)+\phi\right)
$$

i.e. traveling waves with velocity

$$
c_{2}=\frac{1}{3}+\frac{4 \pi^{2}}{9} \sqrt{g} U_{2} \eta_{0}^{2} .
$$

A plot of the shape of this wave is represented on Fig. 2.

Similarly for $S$, we obtain traveling waves running backwards $\eta(x, t)=\eta_{0} \cos \left(x+c_{1} t+\phi\right)$ with velocity

$$
c_{1}=1+4 \pi^{2} \sqrt{g} U_{1} \eta_{0}^{2} .
$$

For $T$, we obtain traveling waves running forwards $\eta(x, t)=\eta_{0} \cos \left(4\left(x-c_{3} t\right)+\phi\right)$ with velocity

$$
c_{3}=\frac{1}{2}+\pi^{2} \sqrt{g} U_{3} \eta_{0}^{2}
$$

A linear stability of the points $P, S$ and $T$ shows that these fixed points of the dynamics are elliptic. It means that nearby trajectories will mimic the behavior of the dynamics of these points. In particular, the surface waves near these points are time quasiperiodic waves close to traveling waves. 


\section{B. Dynamics on a point on the segment $(P S)$}

We consider the point which is at the intersection of $C_{+}\left(\mathbb{I}_{+}\right)$and the segment $(P S)$, for a value of $\mathbb{I}_{+} \in\left[0,2 \mathbb{J}_{+}\right]$. This point is such that $b_{3}(0)=0$ and $d b_{3}(0) / d t=0$. From the equation for $b_{3}(t)$, we deduce that $b_{3}(t)=0$ for any time. From the two invariants $\mathbb{I}_{+}$and $\mathbb{J}_{+}$, we have that $N_{1}$ and $N_{2}$ are constant and equal to $N_{1}(t)=\mathbb{I}_{+} / 2$ and $N_{2}(t)=\left(2 \mathbb{I}_{+}-\mathbb{I}_{+}\right) / 6$. The equations of motion become:

$$
\begin{aligned}
& \frac{d b_{1}}{d t}=-i\left(1+\left(U_{1}-\frac{V_{21}}{6}\right) \mathbb{I}_{+}+\frac{V_{21}}{3} \mathbb{J}_{+}\right) b_{1}, \\
& \frac{d b_{2}}{d t}=-i\left(3+\left(-\frac{U_{2}}{3}+\frac{V_{21}}{2}\right) \mathbb{I}_{+}+\frac{2}{3} V_{21} \mathbb{J}_{+}\right) b_{2} .
\end{aligned}
$$

We deduce the expression of $b_{1}$ and $b_{2}$ :

$$
\begin{aligned}
b_{1}(t) & =\sqrt{\frac{\mathbb{I}_{+}}{2}} \exp \left(-i\left[1+\left(U_{1}-\frac{V_{21}}{6}\right) \mathbb{I}_{+}+\frac{V_{21}}{3} \mathbb{J}_{+}\right] t+\alpha_{1}\right), \\
& =\sqrt{\frac{\mathbb{I}_{+}}{2}} \exp \left[-i\left(c_{1} t+\alpha_{1}\right)\right], \\
b_{2}(t) & =\sqrt{\left(2 \mathbb{J}_{+}-\mathbb{I}_{+}\right) / 6} \exp \left(-i\left[3+\left(-\frac{U_{2}}{3}+\frac{V_{21}}{2}\right) \mathbb{I}_{+}+\frac{2}{3} V_{21} \mathbb{J}_{+}\right] t+\alpha_{2}\right), \\
& =\sqrt{\frac{2 \mathbb{J}_{+}-\mathbb{I}_{+}}{6}} \exp \left[-i\left(9 c_{2} t+\alpha_{2}\right)\right],
\end{aligned}
$$

where $c_{1}=1+\left(U_{1}-V_{21} / 6\right) \mathbb{I}_{+}+V_{21} / 3 \mathbb{I}_{+}$and $c_{2}=\left[3+\left(-U_{2} / 3+V_{21}\right) \mathbb{I}_{+}+2 / 3 V_{21} \mathbb{J}_{+}\right] / 9$.

By taking into account Remark 1 of the Appendix, we have $a_{j}=b_{j}$ for $j=1,2,3$. The expression of the shape of the wave is:

$$
\begin{aligned}
\eta(x, t)= & \frac{g^{-1 / 4}}{2 \pi}\left(\sqrt{\mathbb{I}_{+}} \cos \left(x+c_{1} t+\alpha_{1}\right)\right. \\
& \left.+\sqrt{2 \mathbb{I}_{+}-\mathbb{I}_{+}} \cos \left(9\left(x-c_{2} t\right)+\alpha_{2}\right)\right),
\end{aligned}
$$

which is the sum of two traveling waves with two different velocities.

We notice that on $P$ (respectively on $S$ ), the amplitude of the traveling wave with velocity $c_{1}$ (respectively $c_{2}$ ) vanishes. Therefore the wave associated with $P$ (respectively $S$ ) becomes the traveling wave with velocity $c_{2}$ (respectively $c_{1}$ ) obtained in Sec. III. 


\section{Dynamics of orbits on $C_{+}\left(\mathbb{I}_{+}\right)$}

Next, we extend the dynamics with one resonance, by studying the dynamics on the full sets $C_{+}\left(\mathbb{I}_{+}\right)$for $\mathbb{I}_{+} \in\left[0,2 \mathbb{J}_{+}\right]$. We determine numerically the profiles of the waves which are similar to those obtained previously namely stationary, traveling, time periodic and quasiperiodic waves. This study gives also all the information about the exchanges of energy which occur between the modes.

In order to study the orbits in this case, since we have a Hamiltonian with three degrees of freedom and three constants of motion, we want to perform a canonical change of variables reducing the dimension of the system to one degree of freedom. Furthermore, we would like to change canonically the coordinates into action-angle variables $\left(N_{j}, \Phi_{j}\right)$ such that $b_{j}=\sqrt{N_{j}} e^{-i \Phi_{j}}$.

First we notice that if $N_{3}(0)=0$, then $N_{3}(t)=0$ at any time $\mathrm{t}$ (see Sec. IIIB). In what follows, we always exclude this point which is at the intersection of $(P S)$ and $C_{+}\left(\mathbb{I}_{+}\right)$.

In the case where $\mathbb{I}_{+}=\mathbb{J}_{+} / 2$, we notice that since $\mathbb{K}_{+}=N_{1}-N_{2}$ is a conserved quantity (equal to zero in this case), if $N_{1}$ vanishes at some time, then $N_{2}$ also vanishes. This dynamics occurs at the point $T$ (see Sec. [IIA). Except this particular case, there are two interesting cases where $\mathbb{I}_{+}>\mathbb{I}_{+} / 2$ and $\mathbb{I}_{+}<\mathbb{J}_{+} / 2$. If $\mathbb{I}_{+}<\mathbb{J}_{+} / 2$, only $N_{1}$ vanishes since $N_{2} \geq\left(\mathbb{J}_{+}-2 \mathbb{I}_{+}\right) / 3>0$. If $\mathbb{I}_{+}>\mathbb{I}_{+} / 2$, only $N_{2}$ vanishes since $N_{1} \geq\left(2 \mathbb{I}_{+}-2 \mathbb{J}_{+}\right) / 3>0$.

We remark that $N_{1}$ is such that $\max \left(0, \mathbb{K}_{+}\right) \leq \mathrm{N}_{1} \leq \mathbb{I}_{+} / 2$, so the accessible phase space in the $\left(p_{1}, q_{1}\right)=\left(\operatorname{Re}\left(b_{1}\right), \operatorname{Im}\left(b_{1}\right)\right)$-plane is a circle for $\mathbb{K}_{+}<0$ and an annulus for $\mathbb{K}_{+}>0$. In order to avoid the two cases $N_{1}=0$ or $N_{2}=0$ for which the angle $\phi_{1}$ or $\phi_{2}$ is not defined in the change of variables, we first show that there is only one trajectory $\mathcal{T}_{1}$ such that $N_{1}(t)=0$, i.e which passes by zero in the $\left(p_{1}, q_{1}\right)$ - plane.

First we notice that Hamilton's equations associated with Hamiltonian (12) are invariant by the two transformations:

$$
\begin{aligned}
& \hat{b}_{1}=b_{1}, \\
& \hat{b}_{2}=b_{2} e^{-i \phi}, \\
& \hat{b}_{3}=b_{3} e^{-i \phi / 2},
\end{aligned}
$$


and

$$
\begin{aligned}
& \hat{b}_{1}=b_{1} e^{-i \phi}, \\
& \hat{b}_{2}=b_{2}, \\
& \hat{b}_{3}=b_{3} e^{-i \phi / 2},
\end{aligned}
$$

where $\phi$ is constant. Using these two invariants, one can show that there is basically one trajectory $\mathcal{T}_{1}$ which passes by zero in the $\left(p_{1}, q_{1}\right)$-plane. The closed trajectory $\mathcal{T}_{1}$ divides the phase space in the $\left(p_{1}, q_{1}\right)$-plane into two parts: the inside and the outside of $\mathcal{T}_{1}$.

Similarly, for $\mathbb{K}_{+}>0$, there is only one trajectory $\mathcal{T}_{2}$ which passes by zero in $\left(p_{2}, q_{2}\right)$-plane. We notice that $N_{2}=0$ corresponds to $N_{1}=\mathbb{K}_{+}$and so a trajectory which passes by zero in the $\left(p_{2}, q_{2}\right)$-plane, is a trajectory which passes by the point $N_{1}=\mathbb{K}_{+}$in the $\left(p_{1}, q_{1}\right)$-plane, which is the inside border of the accessible region.

The trajectory $\mathcal{T}_{2}$ divides the annulus into two parts: the inside and the outside of $\mathcal{T}_{2}$. Thus in any of these two parts, $N_{1}$ and $N_{2}$ are strictly positive.

In the regions where all actions $N_{1}, N_{2}$ and $N_{3}$ are strictly positive, we perform a canonical transformation that expresses Hamiltonian (12) into action-angle variables :

$$
b_{j}=\sqrt{N_{j}} e^{-i \Phi_{j}}
$$

This transformation is canonical i.e. :

$$
\sum_{j=1,2,3} i d b_{j} \wedge d b_{j}^{*}=\sum_{j=1,2,3} d N_{j} \wedge d \Phi_{j}
$$

The actions $N_{1}, N_{2}, N_{3}$ are respectively conjugate to the angles $\Phi_{1}, \Phi_{2}, \Phi_{3}$. Hamiltonian (12) becomes

$$
\begin{aligned}
H= & N_{1}+3 N_{2}+2 N_{3}+U_{1} N_{1}^{2}+U_{2} N_{2}^{2}+U_{3} N_{3}^{2} \\
& +V_{21} N_{1} N_{2}-V_{31} N_{1} N_{3}+V_{23} N_{2} N_{3} \\
& +W \sqrt{N_{1} N_{2}} N_{3} \cos \left(\Phi_{1}+\Phi_{2}-2 \Phi_{3}\right) .
\end{aligned}
$$

Hamilton's equations for Hamiltonian (18) are given by

$$
\begin{array}{r}
\frac{d \Phi_{j}}{d t}=\frac{\partial H}{\partial N_{j}}, \\
\frac{d N_{j}}{d t}=-\frac{\partial H}{\partial \Phi_{j}},
\end{array}
$$


for $j=1,2,3$.

Using the invariants $\mathbb{I}_{+}$and $\mathbb{J}_{+}$, we now proceed to the reduction of the dimension of the system to one degree of freedom.

We perform the following canonical transformation from $\left(N_{1}, N_{2}, N_{3}, \Phi_{1}, \Phi_{2}, \Phi_{3}\right)$ to $\left(\tilde{N}_{1}, \mathbb{K}_{+}, \mathbb{I}_{+}, \theta_{1}, \theta_{2}, \theta_{3}\right)$ :

$$
\begin{aligned}
\tilde{N}_{1} & =N_{1}, \\
\mathbb{K}_{+} & =N_{1}-N_{2}, \\
\mathbb{I}_{+} & =2 N_{1}+N_{3}, \\
\theta_{1} & =\Phi_{1}+\Phi_{2}-2 \Phi_{3}, \\
\theta_{2} & =-\Phi_{2}, \\
\theta_{3} & =\Phi_{3} .
\end{aligned}
$$

The actions $\tilde{N}_{1}, \mathbb{K}_{+}$and $\mathbb{I}_{+}$are now respectively conjugate to angles $\theta_{1}, \theta_{2}$ and $\theta_{3}$. In what follows, we use $N_{1}$ instead of $\tilde{N}_{1}$ for simplicity. Hamiltonian (18) becomes

$$
H=\Omega_{0}+\Omega_{1} N_{1}+\Omega_{2} N_{1}^{2}+W \sqrt{N_{1}} \sqrt{N_{1}-\mathbb{K}_{+}}\left(\mathbb{I}_{+}-2 N_{1}\right) \cos \theta_{1}
$$

where the functions $\Omega_{0}$ and $\Omega_{1}$ only depend on the actions $\mathbb{I}_{+}, \mathbb{K}_{+}$, and $\Omega_{2}$ is constant :

$$
\begin{aligned}
& \Omega_{0}=-3 \mathbb{K}_{+}+2 \mathbb{I}_{+}+U_{3} \mathbb{I}_{+}^{2}+U_{2} \mathbb{K}_{+}^{2}-V_{23} \mathbb{I}_{+} \mathbb{K}_{+}, \\
& \Omega_{1}=-2 U_{2} \mathbb{K}_{+}-4 U_{3} \mathbb{I}_{+}-V_{21} \mathbb{K}_{+}-V_{31} \mathbb{I}_{+}+V_{23}\left(\mathbb{I}_{+}+2 \mathbb{K}_{+}\right), \\
& \Omega_{2}=U_{1}+U_{2}+4 U_{3}+V_{21}+2 V_{31}-2 V_{23} .
\end{aligned}
$$

Numerically $\Omega_{2}$ is given by $\Omega_{2}=-3.9642$.

We notice that Hamiltonian (20) does not depend on the angles $\theta_{2}$, and $\theta_{3}$ which is equivalent to saying that $\mathbb{I}_{+}$and $\mathbb{K}_{+}$are invariant (constants of motion).

In order to visualize the dynamics, we perform numerical integration of the equations of motion associated with Hamiltonian (20).

A plot of some trajectories for $\mathbb{J}_{+}=1$ and different values of $\mathbb{I}_{+}$in the accessible region of the phase space (grey region) is given in Fig. 3. In these plots, we have transformed from 
action-angle $\left(N_{1}, \theta_{1}\right)$ to cartesian coordinates $P_{1}=\sqrt{N_{1}} \cos \theta_{1}, Q_{1}=\sqrt{N_{1}} \sin \theta_{1}$. In these last variables, the accessible region is, for $\mathbb{K}_{+}<0$, i.e. $\mathbb{I}_{+}<\mathbb{J}_{+} / 2$, the set of points in the interior of the circle $N_{1}=\mathbb{I}_{+} / 2$ and such that these points are inside $\mathcal{T}_{1}$ or outside $\mathcal{T}_{1}$ (where the canonical transformation from the original variables $b_{j}$ to action-angle variables $\left(N_{j}, \theta_{j}\right)$ is well defined). For $\mathbb{K}_{+}>0$, i.e. $\mathbb{I}_{+}>\mathbb{J}_{+} / 2$, the accessible region is the annulus $\mathbb{K}_{+}<N_{1}<\mathbb{I}_{+} / 2$ and such that these points are inside $\mathcal{T}_{2}$ or outside $\mathcal{T}_{2}$.

We clearly see that these plots are characterized by elliptic, hyperbolic points and the associated separatrices. Below, we discuss the location of these points with respect to the parameter $\mathbb{I}_{+}$.

The fixed points are obtained when $\dot{N}_{1}=0$ and $\dot{\theta}_{1}=0$. As depicted in Fig. 1, there is three families of fixed points. The first family, denoted $e\left(\mathbb{I}_{+}\right)$, is located at $\theta_{1}=0$ and is elliptic. Its position $N_{1}$ is solution of the equation:

$$
\Omega_{1}+2 \Omega_{2} N_{1}+\frac{W}{2}\left[\left(\sqrt{\frac{N_{1}-\mathbb{K}_{+}}{N_{1}}}+\sqrt{\frac{N_{1}}{N_{1}-\mathbb{K}_{+}}}\right)\left(\mathbb{I}_{+}-2 N_{1}\right)-4 \sqrt{N_{1}\left(N_{1}-\mathbb{K}_{+}\right)}\right]=0 .
$$

which is the equation of $\dot{\theta}_{1}=0$ for $\theta_{1}=0$.

There exist two others families of fixed points located at $\theta_{1}=\pi$, one elliptic point denoted $e_{1}\left(\mathbb{I}_{+}\right)$and the other hyperbolic point denoted $h_{1}\left(\mathbb{I}_{+}\right)$.

The location $N_{1}$ is given by the equation:

$$
\Omega_{1}+2 \Omega_{2} N_{1}-\frac{W}{2}\left[\left(\sqrt{\frac{N_{1}-\mathbb{K}_{+}}{N_{1}}}+\sqrt{\frac{N_{1}}{N_{1}-\mathbb{K}_{+}}}\right)\left(\mathbb{I}_{+}-2 N_{1}\right)-4 \sqrt{N_{1}\left(N_{1}-\mathbb{K}_{+}\right)}\right]=0 .
$$

which is the equation of $\dot{\theta}_{1}=0$ for $\theta_{1}=\pi$.

The location of all these fixed points as a function of $\mathbb{I}_{+}$is depicted on Fig. 1 .

We see on the different plots of Fig. 3, that when $\mathbb{I}_{+}$is close to zero, there is no fixed points. When we increase $\mathbb{I}_{+}$there is only one elliptic fixed point $e\left(\mathbb{I}_{+}\right)$( Fig. 3(a) ) which appears near zero. As the value of $\mathbb{I}_{+}$increases, there are three fixed points: besides the previous elliptic fixed point $e\left(\mathbb{I}_{+}\right)$, there is a bifurcation (fold, which is structurally stable) where two

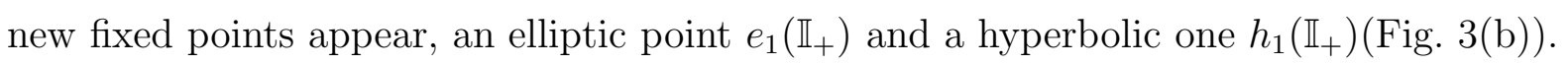

We have a homoclinic connection for the hyperbolic fixed point. When the value of $\mathbb{I}_{+}$ still increases and remains lower than $\mathbb{J}_{+} / 2$, the elliptic fixed point $e\left(\mathbb{I}_{+}\right)$moves to the right and the hyperbolic one $h_{1}\left(\mathbb{I}_{+}\right)$moves to the left, both going towards the border of the phase 
space. At the same time, the elliptic fixed point $e_{1}\left(\mathbb{I}_{+}\right)$moves to the right going to the center of the phase space so approaching $N_{1}=0$. When $\mathbb{I}_{+}$is close to $\mathbb{J}_{+} / 2$, the elliptic fixed point $e_{1}\left(\mathbb{I}_{+}\right)$remains near the border $N_{1}=0$ but it is not apparent in the figures and the hyperbolic one $h_{1}\left(\mathbb{I}_{+}\right)$remains near the border $N_{1}=\mathbb{I}_{+} / 2$. (Fig. $3(\mathrm{c})$ ).

For $\mathbb{I}_{+}>\mathbb{J}_{+} / 2$ the hyperbolic point $h_{1}\left(\mathbb{I}_{+}\right)$remains at the border $N_{1}=\mathbb{I}_{+} / 2$ and the elliptic point $e_{1}\left(\mathbb{I}_{+}\right)$is in the border of the annulus where $\mathbb{K}_{+}>0$. At the same time the region in which $N_{1}$ evolves decreases ( Fig. 匇(d) and Fig. 3(e)). For $\mathbb{I}_{+}$near $2 \mathbb{J}_{+}$, the phase space merges to the point $S$.

We notice that for $\mathbb{I}_{+}=\mathbb{J}_{+} / 2$, the resonance occupies a larger region of phase space than the other cases. This is in agreement with Fig. 11. All these fixed points correspond to periodic variations in variables $N_{1}, N_{2}, N_{3}$ and $\Phi_{1}, \Phi_{2}, \Phi_{3}$. Indeed, due to the invariants given by Eqs.(9)-(10), if $N_{1}$ is fixed, $N_{2}$ and $N_{3}$ remain fixed and the angles $\Phi_{1}, \Phi_{2}, \Phi_{3}$ evolve linearly in time. For example in the case $\mathbb{I}_{+}=\mathbb{J}_{+} / 2$ ( i.e. $\mathbb{K}_{+}=0$ ), we give explicitly the dynamics corresponding to $e\left(\mathbb{J}_{+} / 2\right)$. The equations of motion are

$$
\begin{aligned}
& \frac{d N_{1}}{d t}=W N_{1}\left(\mathbb{I}_{+}-2 N_{1}\right) \sin \theta_{1} \\
& \frac{d \theta_{1}}{d t}=\Omega_{1}+W \mathbb{I}_{+} \cos \theta_{1}+2 N_{1}\left(\Omega_{2}-2 W \cos \theta_{1}\right) .
\end{aligned}
$$

For $\theta_{1}=0$ and

$$
N_{1}=\frac{\Omega_{1}+W \mathbb{I}_{+}}{-2 \Omega_{2}+4 W}
$$

we get an elliptic fixed point with $N_{1} \leq \mathbb{I}_{+} / 2$ for all $\mathbb{I}_{+}=\mathbb{J}_{+} / 2$. This means that this fixed point exists for all admissible values of the parameter $\mathbb{J}_{+}$and for $\mathbb{I}_{+}=\mathbb{J}_{+} / 2$.

We deduce the expressions of the linear motion of the angles :

$$
\begin{aligned}
\Phi_{1}(t) & =\left[1-V_{31} \mathbb{I}_{+}+\frac{1}{2} W \mathbb{I}_{+}+\left(2 U_{1}+V_{21}-2 V_{31}-W\right) N_{1}\right] t+\Phi_{1}(0), \\
& =c_{1} t+\alpha_{1}, \\
\Phi_{2}(t) & =\left[3+V_{23} \mathbb{I}_{+}+\frac{1}{2} W \mathbb{I}_{+}+\left(V_{21}+2 U_{2}-2 V_{23}-W\right) N_{1}\right] t+\Phi_{2}(0), \\
& =9 c_{2} t+\alpha_{2}, \\
\Phi_{3}(t) & =\left[2+2 U_{3} \mathbb{I}_{+}+\left(-V_{31}+V_{23}-4 U_{3}+W\right) N_{1}\right] t+\Phi_{3}(0), \\
& =4 c_{3} t+\alpha_{3} .
\end{aligned}
$$


This linear dynamics gives the following shape of the wave:

$$
\begin{aligned}
\eta(x, t)= & \frac{g^{-1 / 4}}{\pi \sqrt{2}}\left(\sqrt{N_{1}} \cos \left(x+c_{1} t+\alpha_{1}\right)\right. \\
& +\sqrt{3} \sqrt{N_{1}} \cos \left[9\left(x-c_{2} t\right)+\alpha_{2}\right] \\
& \left.+\sqrt{2} \sqrt{\mathbb{I}_{+}-2 N_{1}} \cos \left[4\left(x-c_{3} t\right)+\alpha_{3}\right]\right),
\end{aligned}
$$

which is a sum of three traveling waves with different velocities.

For this case, we have plotted on Fig. 4 the elevation of the surface $\eta(x, t)$. We see that $\eta$ is quasiperiodic in time.

\section{Exchanges of energy on $C_{+}\left(\mathbb{I}_{+}\right)$}

We will now see how the exchanges of energy occur among the three modes for different values of $\mathbb{I}_{+}$.

Since all the orbits of $C_{+}\left(\mathbb{I}_{+}\right)$are closed, the function $N_{1}(t)$ is periodic. From the two invariants $\mathbb{I}_{+}$and $\mathbb{J}_{+}$, the two other actions $N_{2}(t)$ and $N_{3}(t)$ are periodic with the same period as $N_{1}(t)$. Therefore, all the exchanges which occur in the system are periodic in time.

- We consider $C_{+}\left(\mathbb{I}_{+}\right)$with $\frac{\mathbb{J}_{+}}{2}<\mathbb{I}_{+}<2 \mathbb{J}_{+}$. Let us first consider the trajectories with initial conditions close to $S$, i.e., such that $N_{2}(0)$ and $N_{3}(0)$ are of order $\varepsilon<<1$. If we set $\mathbb{I}_{+}=2 \mathbb{I}_{+}-\varepsilon$, we get from Eqs. (9) $-(10)$

$$
\begin{aligned}
& \frac{\mathbb{I}_{+}}{2}-\frac{\varepsilon}{6} \leq N_{1}(t) \leq \frac{\mathbb{I}_{+}}{2}, \\
& 0 \leq N_{2}(t) \leq \frac{\varepsilon}{6}, \\
& 0 \leq N_{3}(t) \leq \frac{\varepsilon}{3},
\end{aligned}
$$

for all $t$.

In fact, this behavior is valid for all values of $\mathbb{I}_{+}$. So, if we have initially $N_{1}(0)>>N_{2}(0)$ and $N_{3}(0)$, then by looking at the invariant $\mathbb{I}_{+}$, we see that for $N_{3}(0)$ close to zero, $N_{1}(t)$ decreases and at the same time $N_{3}(t)$ increases. The invariant $2 N_{2}+N_{3} \approx 2 N_{2}(0)$ shows that $N_{2}(t)$ also decreases and $N_{3}(t)$ does not grow more than $2 N_{2}(0)$ which is small as we can see in Fig. 5, where we have plotted the time evolution of the 
amplitudes $N_{1}, N_{2}$ and $N_{3}$ for the initial condition $\left(N_{1}, \theta_{1}\right)=\left(\frac{\mathbb{I}_{+}}{2}, \pi\right)$.

Therefore, when almost all the energy is initially carried out by the lower frequency mode $N_{1}$, the oscillations of the amplitudes are very small and there is no significant energy exchange between the modes.

By looking directly at Fig. 1, we also see that, for such values of $\mathbb{I}_{+}$, only the modes $N_{2}$ and $N_{3}$ may exchange their energy while $N_{1}$ may only sustain small energy fluctuations. In the same way, for an orbit corresponding to $C_{+}\left(\mathbb{I}_{+}\right)$with $0<\mathbb{I}_{+}<\mathbb{J}_{+} / 2$, there is no significant energy exchanges for initial conditions close to $P$, and in any case only $N_{1}$ and $N_{3}$ enable some energy exchange among the modes.

- In order to obtain large energy exchanges between the three modes, we have to put as much energy in the side bands as in the central carrier: the contribution of energy to the side bands is equal to $\omega_{1} N_{1}+\omega_{2} N_{2}=N_{1}+3 N_{2}$. The maximum energy exchange is obtained when $N_{1}+3 N_{2}=2 N_{3}=\mathbb{J}_{+} / 2$. This happens in the zone where $N_{1}$ and $N_{2}$ are equally important namely close to $C_{+}\left(\mathbb{J}_{+} / 2\right)$.

In this case, using the form of the three integrals of the motion and assuming $\mathbb{I}_{+}=$ $\mathbb{J}_{+} / 2+\varepsilon$, we get:

$$
0 \leq N_{3}(t) \leq \frac{\mathbb{J}_{+}}{2}+\varepsilon, \quad \frac{2 \varepsilon}{3} \leq N_{1}(t) \leq \frac{\mathbb{J}_{+}}{4}+\frac{\varepsilon}{2}
$$

Then if we assume that at $t=0 N_{3}(0)>>N_{1}(0)$ and $N_{2}(0)$ such that the most energy is stored in the highest frequency mode, from $2 N_{1}+N_{3} \approx N_{3}(0)$ and $N_{1}(t)-N_{2}(t)=$ $N_{1}(0)-N_{2}(0)$ we see that $N_{3}(t)$ decreases and at the same time $N_{1}(t)$ and $N_{2}(t)$ increase. So nothing prohibits that energy will be completely transferred from the highest frequency mode into the low frequency modes. What we observe is a periodic modulation of the wave amplitudes. We see that most of the initial energy carried out by the central frequency mode of the wave, $N_{3}$, is transferred to the side bands and the magnitude of the amplitude oscillations is very large. This is the usual Benjamin-Feir instability: the carrier and the two side-bands evolve on a periodic cycle of modulation and demodulation known as Fermi-Pasta-Ulam oscillations. In Fig. 6 we see how the energy exchange is done among the amplitudes $N_{1}, N_{2}$ and $N_{3}$ for for initial $\left(N_{1}, \theta_{1}\right)=\left(\mathbb{I}_{+} / 8,0\right)$.

For this initial condition, we have plotted in Fig. 7 , the shape of the wave $\eta(x, t)$ for 
this initial condition. The periodic modulation and demodulation of the three modes give a shape of the wave which is a modulated traveling wave.

- We can also see exchanges of energy for $\mathbb{I}_{+}=\mathbb{J}_{+} / 2$ i.e. on the segment $C_{+}\left(\mathbb{J}_{+} / 2\right)$ and for initial conditions so that $N_{3}(0)$ is close to zero and $N_{1}, N_{2}$ lies on $C_{+}\left(\mathbb{J}_{+} / 2\right)$ so that $N_{1}(0)=N_{2}(0) \approx \mathbb{J}_{+} / 4$. We recall that if $N_{1}(0)=N_{2}(0)$ then $N_{1}(t)=N_{2}(t)$ and $\mathbb{K}_{+}=0$.

For this case, from Eq.(9), we conclude that $N_{1}(t)$ and $N_{2}(t)$ decrease and $N_{3}(t)$ grows until it reaches its maximum which is $\mathbb{J}_{+} / 4$. As shown in Fig. 8, the trajectory stays a long time close to its initial position and then make a fast excursion before an equally fast return to the departure point. The fast oscillation corresponds to an important exchange of energy between the carrier and the side bands since the carrier almost reaches its maximal energy during the burst. We see in the Fig. 9 the shape of the wave in this case which is quasiperiodic.

\section{DYNAMICS WITH TWO RESONANCES}

In this section we analyze the dynamics of Hamiltonian (8) with the two resonances. The phase space is now the product of $\mathbb{E}_{+}$by $\mathbb{E}_{-}$associated with each resonance. In this case, the dynamics shows a rich variety of regimes allowing a more efficient energy transfer among the modes. We give the dynamics of different orbits in the phase space for different values of the parameters and also give the type of the shape of the wave $\eta(x, t)$.

As in the case of one resonance, we start by simple situations: we explore the dynamics of the points $P_{+} \times P_{-}, S_{+} \times S_{-}, T_{+} \times T_{-}$and the segment $\left(P_{+} S_{+}\right) \times\left(P_{-} S_{-}\right)$for which we get the analytical expression of the wave $\eta(x, t)$.

\section{A. Dynamics on points $P_{+} \times P_{-}, S_{+} \times S_{-}$and $T_{+} \times T_{-}$}

Let us start with the case where the position of the projection of the orbits on $\left(\mathbb{E}_{+}\right) \times\left(\mathbb{E}_{-}\right)$ is fixed at $P_{+} \times P_{-}$which correspond to $\mathbb{I}_{+}=\mathbb{I}_{-}=0$ (see Fig. 1). Since $\mathbb{I}_{+}=2 N_{1}+N_{3}$ is a conserved quantity and $N_{1}, N_{3}$ are such that $N_{1} \geq 0$ and $N_{3} \geq 0$, we have $N_{1}=N_{3}=0$ for all time and $N_{2}=\mathbb{J}_{+} / 3$. Similarly we have $N_{-1}=N_{-3}=0$ and $N_{-2}=\mathbb{J}_{+} / 3$. 
Then for $P_{+} \times P_{-}$, all the actions are constant and the dynamics is connected to the evolution of the angles. The corresponding dynamical equations are:

$$
\begin{gathered}
i \frac{d b_{2}(t)}{d t}=\left(3+2 U_{2}\left(N_{2}-2 N_{-2}\right)\right) b_{2}, \\
i \frac{d b_{-2}(t)}{d t}=\left(3+2 U_{2}\left(N_{-2}-2 N_{2}\right)\right) b_{-2} .
\end{gathered}
$$

The solution in $b_{j}(t)$ is then :

$$
\begin{aligned}
b_{2}(t) & =\sqrt{\mathbb{J}_{+} / 3} \exp \left(-i\left(9 c t+\phi_{+}\right)\right), \\
b_{-2}(t) & =\sqrt{\mathbb{J}_{+} / 3} \exp \left(-i\left(9 \alpha c t+\phi_{-}\right)\right), \\
N_{j}(t) & =0, \quad \text { for } \quad j=1,-1,3,-3,
\end{aligned}
$$

where

$$
\alpha=\frac{J_{0}+\mathbb{J}_{-}-2 \mathbb{J}_{+}}{J_{0}+\mathbb{J}_{+}-2 \mathbb{J}_{-}}, \quad c=\frac{1}{3}\left(1+\frac{\mathbb{J}_{+}-2 \mathbb{J}_{-}}{\mathbb{J}_{0}}\right), \quad \text { and } \quad \mathbb{J}_{0}=\frac{9}{2 U_{2}} .
$$

We notice that $\alpha$ is related to $\mathbb{J}_{+}$and $\mathbb{J}_{-}$by

$$
(1+2 \alpha) \mathbb{J}_{-}-(\alpha+2) \mathbb{J}_{+}=(\alpha-1) \mathbb{J}_{0}
$$

The expression of the shape $\eta(x, t)$ is given by:

$$
\eta(x, t)=\frac{g^{-1 / 4}}{2 \pi \sqrt{2}} \sum_{j=1,2,3}\left|k_{j}\right|^{1 / 4}\left(\left(a_{j}+a_{-j}^{*}\right) e^{i k_{j} x}+\left(a_{j}^{*}+a_{-j}\right) e^{-i k_{j} x}\right)
$$

where the variables $a_{j}$, as function of $b_{j}$ are given by (see Remark 2 of the Appendix):

$$
a_{j}=b_{j}+3 \widetilde{V}_{j, j,-j,-j}^{(4)} b_{-j}^{* 2} b_{j}^{*}, \quad \text { for } \quad j=1,2,3 .
$$

Similarly $a_{-j}$ is given by replacing $k_{j}$ by $-k_{j}$ in Eq. (34), and $a_{j}=0$ for $j \neq 2, j \neq-2$. Then we deduce the surface shape:

$$
\begin{aligned}
\eta(x, t)= & \frac{g^{-1 / 4}}{\pi \sqrt{2}}\left[\sqrt{\mathbb{J}_{+}} \cos \left(9(x-c t)-\phi_{+}\right)+\sqrt{\mathbb{J}_{-}} \cos \left(9(x+\alpha c t)+\phi_{-}\right)\right. \\
& +V \mathbb{J}_{-} \sqrt{\mathbb{J}_{+}} \cos \left(9(x+(2 \alpha+1) c t)+2 \phi_{-}+\phi_{+}\right) \\
& \left.+V \mathbb{J}_{+} \sqrt{\mathbb{J}_{-}} \cos \left(9(x-(\alpha+2) c t)-2 \phi_{+}-\phi_{-}\right)\right]
\end{aligned}
$$

where $V=\widetilde{V}_{2,2,-2,-2}^{(4)} \approx-0.082$.

The wave is the sum of four traveling waves with four a priori different velocities 
$c, \alpha c,(2 \alpha+1) c$, and $(\alpha+2) c$.

According to the values of $\mathbb{J}_{+}$and $\mathbb{J}_{-}$and more precisely, depending on $\alpha$, these solutions are fixed points, traveling or standing waves, periodic or quasiperiodic solutions.

- If $\mathbb{J}_{+}=\mathbb{J}_{-}$, then $\alpha=1$. We deduce the shape of the wave :

$$
\begin{aligned}
\eta(x, t)= & \frac{g^{-1 / 4} \sqrt{2}}{\pi} \sqrt{\mathbb{J}_{+}} \cos \left(9 x-\frac{\phi_{+}-\phi_{-}}{2}\right) \\
& \times\left(\cos \left(9 c t+\frac{\phi_{+}+\phi_{-}}{2}\right)+V \mathbb{J}_{+} \cos \left(27 c t+3 \frac{\phi_{+}+\phi_{-}}{2}\right)\right) .
\end{aligned}
$$

which is a standing wave. Such wave is represented in Fig. 10(a).

- For the particular case $\mathbb{J}_{+}=\mathbb{J}_{-}=J_{0}, \alpha$ is not defined and the velocities $c$ and $\alpha c$ vanish. The expression of the surface wave is:

$$
\begin{aligned}
\eta(x, t)= & \frac{g^{-1 / 4} \sqrt{2}}{\pi} \sqrt{\mathbb{J}_{+}}\left(\cos \left(9 x-\frac{\phi_{+}-\phi_{-}}{2}\right)\right. \\
& \times\left(\cos \left(\frac{\phi_{+}+\phi_{-}}{2}\right)+V \mathbb{J}_{+} \cos \left(3 \frac{\phi_{+}+\phi_{-}}{2}\right)\right) .
\end{aligned}
$$

The wave is stationary (see Fig. 10(b)) and its amplitude is a function of the initial phases.

- When $\mathbb{J}_{+}+\mathbb{J}_{-}=2 J_{0}$ so that $\alpha=-1$, we have the unique family of traveling waves with velocity $c$ whose shape is given by:

$$
\begin{aligned}
\eta(x, t)= & \frac{g^{-1 / 4}}{\pi \sqrt{2}}\left[\sqrt{\mathbb{J}_{+}} \cos \left(9(x-c t)-\phi_{+}\right)+\sqrt{\mathbb{J}_{-}} \cos \left(9(x-c t)+\phi_{-}\right)\right. \\
& +V \mathbb{J}_{-} \sqrt{\mathbb{J}_{+}} \cos \left(9(x-c t)+2 \phi_{-}+\phi_{+}\right) \\
& \left.+V \mathbb{J}_{+}{\sqrt{\mathbb{J}_{-}}} \cos \left(9(x-c t)-2 \phi_{+}-\phi_{-}\right)\right] .
\end{aligned}
$$

Fig. 10(c) shows the elevation of this surface.

- Time periodic waves are obtained for $\alpha \in \mathbb{Q}$ including the particular case $-\mathbb{J}_{+}+2 \mathbb{J}_{-}=$ $J_{0}$ and $-\mathbb{J}_{-}+2 \mathbb{J}_{+}=J_{0}$, for which $\alpha$ is respectively indefinite or zero. 
The ratios between the four angles, evolving in time, which are $-c t, \alpha c t,(2 \alpha+1) c t$ and $-(\alpha+2) c t$ are rational. The wave is periodic both in space and time, forming the square-shape patterns as observed in Fig. 10(d).

- In the case where $\alpha$ is irrational, the wave is quasiperiodic in time, expressed as a sum of four traveling waves with different velocities. We have plotted such wave in Fig. 10(e).

We notice that a similar variety of regular surface shapes ( standing, stationary, traveling, time-periodic and quasiperiodic waves) are obtained for the two other points of $\left(\mathbb{E}_{+}\right) \times\left(\mathbb{E}_{-}\right)$ which are $S_{+} \times S_{-}, T_{+} \times T_{-}$, and for the points $P_{+} \times S_{-}, P_{+} \times T_{-}, S_{+} \times T_{-}, S_{+} \times P_{-}$, $T_{+} \times S_{-}$, and $T_{+} \times P_{-}$.

\section{B. Dynamics of a point on the segment $\left(P_{+} S_{+}\right) \times\left(P_{-} S_{-}\right)$}

We consider the point which is at the intersection of $C_{+}\left(\mathbb{I}_{+}\right) \times C_{-}\left(\mathbb{I}_{-}\right)$and $\left(P_{+} S_{+}\right) \times$ $\left(P_{-} S_{-}\right)$. This point is such that $b_{3}(0)=0$ and $b_{-3}(0)=0$. From equations of motion of Hamiltonian (8), we deduce $b_{3}(t)=0$ and $b_{-3}(t)=0$ for any time. From the invariants we obtain that $N_{1}, N_{2}, N_{-1}$ and $N_{-2}$ are constant and equal to $N_{1}(t)=\mathbb{I}_{+} / 2$ and $N_{2}(t)=$ $\left(2 \mathbb{J}_{+}-\mathbb{I}_{+}\right) / 6, N_{-1}(t)=\mathbb{I}_{-} / 2$ and $N_{-2}(t)=\left(2 \mathbb{J}_{-}-\mathbb{I}_{-}\right) / 6$. The equations of motion become:

$$
\begin{aligned}
\frac{d b_{1}}{d t} & =-i\left(1+\left(U_{1}-\frac{V_{21}}{6}\right) \mathbb{I}_{+}-\left(2 U_{1}+\frac{V_{-21}}{6}\right) \mathbb{I}_{-}+\frac{V_{21}}{3} \mathbb{J}_{+}+\frac{V_{-21}}{3} \mathbb{J}_{-}\right) b_{1}, \\
\frac{d b_{2}}{d t} & =-i\left(3+\left(-\frac{U_{2}}{3}+\frac{V_{21}}{2}\right) \mathbb{I}_{+}+\left(\frac{2 U_{2}}{3}+\frac{V_{-21}}{2}\right) \mathbb{I}_{-}+\frac{2}{3} U_{2} \mathbb{J}_{+}-\frac{4}{3} U_{2} \mathbb{J}_{-}\right) b_{2}, \\
\frac{d b_{-1}}{d t} & =-i\left(1-\left(2 U_{1}+\frac{V_{-21}}{6}\right) \mathbb{I}_{+}+\left(U_{1}-\frac{V_{21}}{6}\right) \mathbb{I}_{-}+\frac{V_{-21}}{3} \mathbb{J}_{+}+\frac{V_{21}}{3} \mathbb{J}_{-}\right) b_{-1}, \\
\frac{d b_{-2}}{d t} & =-i\left(3+\left(\frac{2 U_{2}}{3}+\frac{V_{-21}}{2}\right) \mathbb{I}_{+}+\left(-\frac{U_{2}}{3}+\frac{V_{21}}{2}\right) \mathbb{I}_{-}-\frac{4}{3} U_{2} \mathbb{J}_{+}+\frac{2}{3} U_{2} \mathbb{J}_{-}\right) b_{-2} .
\end{aligned}
$$

We deduce the expression of the shape of the wave :

$$
\begin{aligned}
\eta(x, t)= & \frac{g^{-1 / 4}}{2 \pi}\left[\sqrt{\mathbb{I}_{+}} \cos \left(x+c_{1} t+\alpha_{1}\right)+\sqrt{2 \mathbb{J}_{+}-\mathbb{I}_{+}} \cos \left(9\left(x-c_{2} t\right)+\alpha_{2}\right)\right. \\
& \left.+\sqrt{\mathbb{I}_{-}} \cos \left(x+c_{-1} t+\alpha_{-1}\right)+\sqrt{2 \mathbb{J}_{-}-\mathbb{I}_{-}} \cos \left(9\left(x-c_{-2} t\right)+\alpha_{-2}\right)\right],
\end{aligned}
$$


where $c_{1}=1+\left(U_{1}+V_{21} / 6\right) \mathbb{I}_{+}-\left(2 U_{1}+V_{-21} / 6\right) \mathbb{I}_{-}+V_{21} \mathbb{I}_{+} / 3+V_{-21} \mathbb{I}_{-} / 3$,

$c_{2}=\left[3+\left(-U_{2} / 3+V_{21} / 2\right) \mathbb{I}_{+}+\left(2 U_{2} / 3+V_{-21} / 2\right) \mathbb{I}_{-}+2 U_{2} \mathbb{J}_{+} / 3-4 U_{2} \mathbb{J}_{-} / 3\right] / 9$,

$c_{-1}=1-\left(2 U_{1}+V_{-21} / 6\right) \mathbb{I}_{+}+\left(U_{1}+V_{21} / 6\right) \mathbb{I}_{-}+V_{-21} \mathbb{I}_{+} / 3+V_{21} \mathbb{J}_{-} / 3$, and

$c_{-2}=\left[3+\left(2 U_{2} / 3+V_{-21} / 2\right) \mathbb{I}_{+}+\left(-U_{2} / 3+V_{21} / 2\right) \mathbb{I}_{-}-4 U_{2} \mathbb{J}_{+} / 3+2 U_{2} \mathbb{J}_{-} / 3\right] / 9$.

This wave is the sum of four traveling waves with four different velocities.

\section{Dynamics of orbits on the segment $\left(P_{+} S_{+}\right)$times the interior of $C_{-}\left(\mathbb{I}_{-}\right)$}

We consider the dynamics of $\left(P_{+} S_{+}\right)$times the interior of $C_{-}\left(\mathbb{I}_{-}\right)$(or similarly the interior of $C_{+}\left(\mathbb{I}_{+}\right)$times $\left.\left(P_{-} S_{-}\right)\right)$. We have $b_{3}(t)=0$ for all $t$, so $N_{3}(t)=0$ is a constant of motion and $N_{1}(t)$ and $N_{2}(t)$ are constant for all t. We have now a system with five degrees of freedom $b_{1}, b_{2}, b_{-1}, b_{-2}$ and $b_{-3}$ with five constants of motion, $\mathbb{I}_{+}, \mathbb{I}_{-}, \mathbb{I}_{+}, \mathbb{I}_{-}$and $H$. It is then integrable. The modes $b_{1}$ and $b_{2}$ evolve with a constant modulus and a phase depending on $\left|b_{-1}\right|,\left|b_{-2}\right|$ and $\left|b_{-3}\right|$. The dynamics of the modes $b_{-1}, b_{-2}$ and $b_{-3}$ in $C_{-}\left(\mathbb{I}_{-}\right)$ depend on the second resonance just through the modulus of $b_{1}$ and $b_{2}$ which are constant. So the exchanges of energy on $C_{-}\left(\mathbb{I}_{-}\right)$are not influenced by the evolution of $b_{1}$ and $b_{2}$. The dynamics of the modes $b_{-1}, b_{-2}$ and $b_{-3}$ is then similar to the one studied in the case with one resonance in Sec. [II].

\section{Dynamics in the interior of $C_{+}\left(\mathbb{I}_{+}\right) \times C_{-}\left(\mathbb{I}_{-}\right)$}

In order to study the dynamics on the nontrivial sets $C_{+}\left(\mathbb{I}_{+}\right) \times C_{-}\left(\mathbb{I}_{-}\right)$on the interior of the phase space $\left(\mathbb{E}_{+}\right) \times\left(\mathbb{E}_{-}\right)$, i.e. such that $N_{j}$ are strictly positive for $j=-1,-2,-3,1,2,3$, we perform a canonical transformation in action-angle coordinates, $b_{j}=\sqrt{N_{j}} e^{-i \Phi_{j}}$ and Hamiltonian (8) becomes:

$$
\begin{aligned}
H= & \sum_{j=1,2,3} \omega_{j}\left(N_{j}+N_{-j}\right)+\sum_{j=1,2,3} U_{j}\left(N_{j}^{2}+N_{-j}^{2}-4 N_{j} N_{-j}\right) \\
& +V_{21}\left(N_{1} N_{2}+N_{-1} N_{-2}\right)+V_{-21}\left(N_{1} N_{-2}+N_{-1} N_{2}\right) \\
& -V_{31}\left(N_{1} N_{3}+N_{-1} N_{-3}\right)+V_{-31}\left(N_{1} N_{-3}+N_{-1} N_{3}\right)
\end{aligned}
$$




$$
\begin{aligned}
& +V_{23}\left(N_{2} N_{3}+N_{-2} N_{-3}\right)-V_{-23}\left(N_{2} N_{-3}+N_{-2} N_{3}\right) \\
& +W \sqrt{N_{1} N_{2}} N_{3} \cos \left(\Phi_{1}+\Phi_{2}-2 \Phi_{3}\right) \\
& +W \sqrt{N_{-1} N_{-2}} N_{-3} \cos \left(\Phi_{-1}+\Phi_{-2}-2 \Phi_{-3}\right) .
\end{aligned}
$$

Using the invariants $\mathbb{I}_{+}, \mathbb{I}_{-}, \mathbb{J}_{+}$and $\mathbb{J}_{-}$, we now proceed to the reduction of the dimension of the system to two degrees of freedom.

We perform as in the case with one resonance the canonical transformation from $\left(N_{1}, N_{2}, N_{3}, N_{-1}, N_{-2}, N_{-3}, \Phi_{1}, \Phi_{2}, \Phi_{3}, \Phi_{-1}, \Phi_{-2}, \Phi_{-3}\right)$ to $\left(\tilde{N}_{1}, \mathbb{K}_{+}, \mathbb{I}_{+}, \tilde{N}_{-1}, \mathbb{K}_{-}, \mathbb{I}_{-}, \theta_{1}, \theta_{2}, \theta_{3}, \theta_{-1}, \theta_{-2}, \theta_{-3}\right)$ :

$$
\begin{aligned}
\tilde{N}_{1} & =N_{1}, \\
\mathbb{K}_{+} & =N_{1}-N_{2}, \\
\mathbb{I}_{+} & =2 N_{1}+N_{3}, \\
\theta_{1} & =\Phi_{1}+\Phi_{2}-2 \Phi_{3}, \\
\theta_{2} & =-\Phi_{2}, \\
\theta_{3} & =\Phi_{3}, \\
\tilde{N}_{-1} & =N_{-1}, \\
\mathbb{K}_{-} & =N_{-1}-N_{-2}, \\
\mathbb{I}_{-} & =2 N_{-1}+N_{-3}, \\
\theta_{-1} & =\Phi_{-1}+\Phi_{-2}-2 \Phi_{-3}, \\
\theta_{-2} & =-\Phi_{-2}, \\
\theta_{-3} & =\Phi_{-3} .
\end{aligned}
$$

The actions $\tilde{N}_{1}, \mathbb{K}_{+}, \mathbb{I}_{+}, \tilde{N}_{-1}, \mathbb{K}_{-}$and $\mathbb{I}_{-}$are now respectively conjugate to angles $\theta_{1}, \theta_{2}$, $\theta_{3}, \theta_{-1}, \theta_{-2}$, and $\theta_{-3}$. In what follows, we use $N_{1}, N_{-1}$ instead of $\tilde{N}_{1}$ and $\tilde{N}_{-1}$ for simplicity. Hamiltonian (38) becomes:

$$
\begin{aligned}
H= & \Omega_{0}+\Omega_{1} N_{1}+\Omega_{-1} N_{-1}+\Omega_{2}\left(N_{1}^{2}+N_{-1}^{2}\right)+\Omega_{3} N_{1} N_{-1} \\
& +W \sqrt{N_{1}} \sqrt{N_{1}-\mathbb{K}_{+}}\left(\mathbb{I}_{+}-2 N_{1}\right) \cos \theta_{1} \\
& +W \sqrt{N_{-1}} \sqrt{N_{-1}-\mathbb{K}_{-}}\left(\mathbb{I}_{-}-2 N_{-1}\right) \cos \theta_{-1}
\end{aligned}
$$


where the functions $\Omega_{0}, \Omega_{1}$ and $\Omega_{-1}$ only depend on the actions $\mathbb{I}_{+}, \mathbb{K}_{+}, \mathbb{I}_{-}, \mathbb{K}_{-}$and $\Omega_{2}$ and $\Omega_{3}$ are constant :

$$
\begin{aligned}
\Omega_{0}= & -3\left(\mathbb{K}_{-}+\mathbb{K}_{+}\right)+2\left(\mathbb{I}_{-}+\mathbb{I}_{+}\right)+U_{3}\left(\mathbb{I}_{-}^{2}+\mathbb{I}_{+}^{2}-4 \mathbb{I}_{+} \mathbb{I}_{-}\right) \\
& +U_{2}\left(\mathbb{K}_{-}^{2}+\mathbb{K}_{+}^{2}-4 \mathbb{K}_{+} \mathbb{K}_{-}\right) \\
& -V_{23}\left(\mathbb{I}_{+} \mathbb{K}_{+}+\mathbb{I}_{-} \mathbb{K}_{-}\right)+V_{-23}\left(\mathbb{I}_{+} \mathbb{K}_{-}+\mathbb{I}_{-} \mathbb{K}_{+}\right), \\
\Omega_{1}= & 2 U_{2}\left(-\mathbb{K}_{+}+2 \mathbb{K}_{-}\right)+4 U_{3}\left(-\mathbb{I}_{+}+2 \mathbb{I}_{-}\right)-V_{21} \mathbb{K}_{+}-V_{-21} \mathbb{K}_{-} \\
& -V_{31} \mathbb{I}_{+}+V_{-31} \mathbb{I}_{-}+V_{23}\left(\mathbb{I}_{+}+2 \mathbb{K}_{+}\right)-V_{-23}\left(\mathbb{I}_{-}+2 \mathbb{K}_{-}\right), \\
\Omega_{-1}= & 2 U_{2}\left(-\mathbb{K}_{-}+2 \mathbb{K}_{+}\right)+4 U_{3}\left(-\mathbb{I}_{-}+2 \mathbb{I}_{+}\right)-V_{21} \mathbb{K}_{-}-V_{-21} \mathbb{K}_{+} \\
& -V_{31} \mathbb{I}_{-}+V_{-31} \mathbb{I}_{+}+V_{23}\left(\mathbb{I}_{-}+2 \mathbb{K}_{-}\right)-V_{-23}\left(\mathbb{I}_{+}+2 \mathbb{K}_{+}\right), \\
\Omega_{2}= & U_{1}+U_{2}+4 U_{3}+V_{21}+2 V_{31}-2 V_{23}, \\
\Omega_{3}= & -4 U_{1}-4 U_{2}-16 U_{3}+2 V_{-21}-4 V_{-31}+4 V_{-23} .
\end{aligned}
$$

Numerically $\Omega_{2}$ and $\Omega_{3}$ are given by: $\Omega_{2}=-3.9642, \Omega_{3}=-16.0933$.

Since Hamiltonian (40) does not depend on $\theta_{2}, \theta_{3}, \theta_{-2}$ and $\theta_{-3}$, we can consider $\mathbb{I}_{+}, \mathbb{K}_{+}, \mathbb{I}_{-}$, $\mathbb{K}_{-}$as parameters of the system for the dynamics of $N_{1}, N_{-1}, \theta_{1}$ and $\theta_{-1}$.

We recall that $N_{1}$ and $N_{-1}$ are such that $\max \left(0, \mathbb{K}_{+}\right)<\mathrm{N}_{1}<\mathbb{I}_{+} / 2$ and $\max \left(0, \mathbb{K}_{-}\right)<\mathrm{N}_{-1}<$ $\mathbb{I}_{-} / 2$. The accessible part of phase space is thus contained into the product of two interiors of a circle or an annulus, depending on the values of $\mathbb{I}_{+}$and $\mathbb{I}_{-}$.

In order to visualize the dynamics on $C_{+}\left(\mathbb{I}_{+}\right) \times C_{-}\left(\mathbb{I}_{-}\right)$we compute Poincaré sections of the dynamics and then we project these surfaces on $\left(N_{1}, \theta_{1}\right)$-plane. The Poincaré sections $\Sigma$ are constructed in the following way:

$$
\Sigma=\left\{\left(N_{1}, N_{-1}, \theta_{1}, \theta_{-1}\right): \theta_{-1}=0, \frac{d \theta_{-1}}{d t}<0, H=H\left(N_{1}, N_{-1}, \theta_{1}, \theta_{-1}, \mathbb{I}_{+}, \mathbb{I}_{-}\right)\right\} .
$$

Now the parameters are the energy $H$, and the four invariants $\mathbb{I}_{+}, \mathbb{I}_{-}, \mathbb{J}_{+}$and $\mathbb{J}_{-}$.

Since Hamiltonian (38) has two degrees of freedom, Poincaré sections are two dimensional and therefore we get an accurate picture of the dynamics by looking at the projections of $\Sigma$ on $\left(N_{1}, \theta_{1}\right)$. For most of the values of the parameters, these sections show a rich variety for the dynamics, a mixed phase space with regular and chaotic behaviors.

We give a plot of some projections for $\mathbb{J}_{+}=\mathbb{J}_{-}=1, H=2$ and for different values of $\mathbb{I}_{+}=\mathbb{I}_{-}:$from $\mathbb{I}_{+}=\mathbb{I}_{-}=0.3$ to $\mathbb{I}_{+}=\mathbb{I}_{-}=0.8$.

We observe on Fig. 11(a), when $\mathbb{I}_{+}$and $\mathbb{I}_{-}$are near zero so that we are in the neighborhood 
of the point $P_{+} \times P_{-}$, the motion is completely regular and all the orbits lie on invariant tori. For this value of $H$, the dynamics occurs close to the center of the accessible region of phase space. The elliptic and hyperbolic fixed points of the Poincaré sections are of the same type as those of the elliptic and hyperbolic orbits of the case with one resonance. Besides these points, there are one elliptic and two hyperbolic points due to the second resonance. We recall that the invariants depend on the variables of only one resonance. So, for small values of $\mathbb{I}_{+}$and $\mathbb{I}_{-}$we can make the same reasoning as for the one resonance case and we can conclude that in the neighborhood of the point $P_{+} \times P_{-}$, the exchanges of energy between the modes are not significant and it leads to a regular behavior of the system. Thus in the system with small values of $\mathbb{I}_{+}$and $\mathbb{I}_{-}$, the only possible motion for the system is periodic or quasi-periodic in time, corresponding to regular shapes for the wave $\eta(x, t)$ (see Sec. [II). To undergo a transition to chaos, we increase the parameter $\mathbb{I}_{+}$( which increases the effect of the nonlinear terms up to a certain threshold), and for a value of $\mathbb{I}_{+}$in the neighborhood of $\mathbb{I}_{+}=\mathbb{J}_{+} / 2$, we see in Fig. 11(b) and Fig. 11(c), that the phase space is made up of regular and chaotic motions. Chaotic trajectories are located between regular trajectories (on invariant tori). We observe that the motion is clearly affected by the vicinity of hyperbolic points: the irregular motion appears in the neighborhood of hyperbolic periodic orbits breaking up their neighboring KAM tori.

The first of the two regular zones which surround the chaotic sea (outer region), is obtained for large amplitudes of the modes of low frequency $N_{1}$ and $N_{-1}$ which are larger than the ones of high frequency modes $N_{3}$ and $N_{-3}$. The second regular zone (inner region) is obtained when the amplitude of the modes of low frequency $N_{1}$ and $N_{-1}$ are small. In these regular zones, the exchanges of energy are not significant and give rise to regular behaviors of the system. On the other hand, for orbits close to the hyperbolic points, the modes oscillate chaotically (see Fig. 12). As the parameter $\mathbb{I}_{+}$increases, the region in which $N_{1}$ evolves shrinks and all the orbits become stable, the system admits only regular behaviors as it can be seen on Fig. 11(d). This is due to the fact that in the accessible region, the values of $N_{1}$ are larger than those of $N_{3}$ and for this case the exchanges among the modes are not significant.

We conclude that chaotic exchanges of energy may be done in the neighborhood of $C_{+}\left(\frac{\mathbb{J}_{+}}{2}\right) \times$ $C_{-}\left(\frac{\mathbb{J}_{-}}{2}\right)$. In Fig. 12, we give the plot of the modes $N_{j}(t), j=1,2,3,-1,-2,-3$ and in Fig. 13, we give the shape of the wave $\eta(x, t)$ for $\mathbb{I}_{+}=\mathbb{I}_{-}=\mathbb{J}_{+} / 2$ and for an initial condition 
$\left(N_{1}, \theta_{1}\right)=(0.152,-3.07)$ which is in the chaotic region. In these two plots, we see the irregular motion of the modes $N_{j}$ (chaotic exchanges of energy), and the chaotic structure of $\eta(x, t)$. We notice that in the chaotic zones, significant energy exchanges occur among the modes.

\section{CONCLUSION}

Following the framework of Zakharov in Ref. [3] and Krazitskii in Ref. [18, we have built a Hamiltonian model for six interacting waves for gravity water waves in infinite depth. The interaction are those of two coupled Benjamin-Feir resonances. The knowledge of four constants of motion allowed us to reduce the system to a Hamiltonian system with two degrees of freedom. We have first studied the dynamical properties of the Hamiltonian with only one resonance which is integrable. This simple case allowed us to understand the basics of the full dynamics. A whole variety of solutions have been found in this simple case such as traveling, time periodic and quasi-periodic waves. We showed that exchanges of energy occur in the zone of phase space where the energies initially put into the two side bands

are equally important and when the energy in the central mode is the same as the sum of the energies of the two side bands. This situation corresponds to phase space regions close to the separatrices. All these exchanges are done in a periodic way. Thus, when we take into account only one resonance, the only possible motion is a regular time quasiperiodic (and space periodic) wave.

Then we have studied the dynamics with the two resonances. Besides the regular waves such as traveling, time periodic and quasiperiodic waves, the full system exhibits stationary and standing waves. We have visualized the dynamics using numerical Poincaré sections. We have showed that the system with the two coupled resonances undergoes a transition to chaos. Chaotic behaviors occur in the regions where the energies of the side bands of each resonance are equally important and near the broken separatrices. In this case, there is a strong exchange of energy between all the modes and it is done in chaotic way. 


\section{DERIVATION OF THE HAMILTONIAN GIVEN BY EQ. (8)}

In this appendix, we briefly derive the expression of Hamiltonian (8) from the Hamiltonian derived by Krazitskii in Ref. 18 given by Eq. (2.11). We get the expression for the Hamiltonian in the case where the three modes (denoted by 1,2 and 3) are such that $k_{1}+k_{2}=2 k_{3}$ and $\omega_{1}+\omega_{2}=2 \omega_{3}$.

In this case, we notice that there are no terms of order 3, i.e. terms of the form $a_{k} a_{k^{\prime}} a_{k^{\prime \prime}}$ such that $k=k^{\prime}+k^{\prime \prime}$ for $k, k^{\prime}, k^{\prime \prime} \in\left\{k_{1}, k_{2}, k_{3},-k_{1},-k_{2},-k_{3}\right\}$.

We expand this Hamiltonian up to order four, i.e. we neglect terms of order five in Hamiltonian (2.11) of Ref. [18] since we are in the case of weak nonlinearity ( small amplitudes of the wave). Hamiltonian (2.11) of Ref. [18 becomes:

$$
\begin{aligned}
H= & \sum_{i=1,2,3} \omega_{i}\left(a_{i} a_{i}^{*}+a_{-i} a_{-i}^{*}\right)+ \\
& +6 \sum_{\substack{i, j=1,2,3, i \neq j}} V_{i, i,-j, j}^{(1)}\left(a_{-i}^{*} a_{-i} a_{-j} a_{j}+a_{i}^{*} a_{i} a_{j} a_{-j}+c . c\right) \\
& +3 V_{2,-1,3,3}^{(1)}\left(a_{2}^{*} a_{-1} a_{3}^{2}+a_{-2}^{*} a_{1} a_{-3}^{2}+c . c\right)+3 V_{1,-2,3,3}^{(1)}\left(a_{1}^{*} a_{-2} a_{3}^{2}+a_{-1}^{*} a_{2} a_{-3}^{2}+c . c\right) \\
& +3 V_{3,-3,1,2}^{(1)}\left(a_{3}^{*} a_{-3} a_{1} a_{2}+a_{-3}^{*} a_{3} a_{-1} a_{-2}+c . c\right) \\
& +\frac{1}{2} \sum_{i=1,2,3} V_{i, i, i, i}^{(2)}\left(a_{i}^{* 2} a_{i}^{2}+a_{-i}^{* 2} a_{-i}^{2}\right)+2 \sum_{i=1,2,3} V_{i,-i, i,-i}^{(2)}\left(a_{i}^{*} a_{-i}^{*} a_{i} a_{-i}\right) \\
& +2 \sum_{\substack{i, j=1,2,3, i \neq j}}\left(V_{i, j, i, j}^{(2)}\left(a_{i}^{*} a_{j}^{*} a_{i} a_{j}+a_{-i}^{*} a_{-j}^{*} a_{-i} a_{-j}\right)+V_{i,-j, i,-j}^{(2)}\left(a_{i}^{*} a_{-j}^{*} a_{i} a_{-j}+a_{-i}^{*} a_{j}^{*} a_{-i} a_{j}\right)\right) \\
& +2 \sum_{\substack{i, j=1,2,3, i \neq j}}^{(2)} V_{-i, i,-j, j}\left(a_{-i}^{*} a_{i}^{*} a_{-j} a_{j}+c . c\right)+V_{1,2,3,3}^{(2)}\left(a_{1}^{*} a_{2}^{*} a_{3}^{2}+a_{-1}^{*} a_{-2}^{*} a_{-3}^{2}+c . c\right) \\
& +2 V_{3,-1,-3,2}^{(2)}\left(a_{3}^{*} a_{-1}^{*} a_{-3} a_{2}+a_{-3}^{*} a_{1}^{*} a_{3} a_{-2}+c . c\right) \\
& +\frac{3}{2} \sum_{\substack{i=1,2,3 \\
-i,-i, i, i}} V_{-i}^{(4)}\left(a_{i}^{* 2} a_{i}^{* 2}+c . c\right)+6 \sum_{\substack{i, j=1,2,3, i \neq j}} V_{-i, i,-j, j}^{(4)}\left(a_{-i}^{*} a_{i}^{*} a_{-j}^{*} a_{j}^{*}+c . c\right) \\
& +3 V_{1,2,-3,-3}^{(4)}\left(a_{-1}^{*} a_{-2}^{*} a_{3}^{* 2}+a_{1}^{*} a_{2}^{*} a_{-3}^{* 2}+c . c\right),
\end{aligned}
$$

where the coefficients verify the symmetry $V_{i, j, k, l}^{(n)}=V_{-i,-j,-k,-l}^{(n)}$, for all $n=1,2,4$ and $i, j, k, l \in\{1,2,3,-1,-2,-3\}$ as well as the other symmetries mentioned in Ref. [18. We verify in the expressions of the coefficients given in Ref. 18 that all the coefficients $V_{i, i,-i, i}^{(1)}$ are equal to zero.

The canonical transformation from the variables $a_{k}$ to the new canonical variables $b_{k}$, 
that maps Hamiltonian (43) to its normal form up to terms of order 5 in $b_{j}$ is given by:

$$
\begin{aligned}
a_{1}= & b_{1}+6 \widetilde{V}_{1,1,-2,2}^{(1)}\left(b_{1} b_{2} b_{-2}+b_{1} b_{2}^{*} b_{-2}^{*}\right)+6 \widetilde{V}_{1,1,-3,3}^{(1)}\left(b_{1} b_{3} b_{-3}+b_{1} b_{3}^{*} b_{-3}^{*}\right) \\
& +6 \widetilde{V}_{2,2,-1,1}^{(1)}\left(b_{-2} b_{-2}^{*} b_{-1}^{*}+b_{2} b_{2}^{*} b_{-1}^{*}\right)+6 \widetilde{V}_{3,3,-1,1}^{(1)}\left(b_{-3} b_{-3}^{*} b_{-1}^{*}+b_{3} b_{3}^{*} b_{-1}^{*}\right) \\
& +3 \widetilde{V}_{2,-1,3,3}^{(1)} b_{-2} b_{-3}^{* 2}+3 \widetilde{V}_{1,-2,3,3}^{(1)} b_{-2} b_{3}^{2}+3 \widetilde{V}_{3,-3,1,2}^{(1)} b_{3} b_{-3}^{*} b_{2}^{*} \\
& +2 \widetilde{V}_{-1,1,-2,2}^{(2)} b_{-1}^{*} b_{-2} b_{2}+2 \widetilde{V}_{-1,1,-3,3}^{(2)} b_{-1}^{*} b_{-3} b_{3}+2 \widetilde{V}_{-1,3,-3,2}^{(2)} b_{3} b_{-3}^{*} b_{-2} \\
& +3 \widetilde{V}_{1,1,-1,-1}^{(4)} b_{-1}^{* 2} b_{1}^{*}+\widetilde{V}_{-1,1,-2,2}^{(4)} b_{-1}^{*} b_{-2}^{*} b_{2}^{*}+\widetilde{V}_{-1,1,-3,3}^{(4)} b_{-1}^{*} b_{-3}^{*} b_{3}^{*} \\
& +3 \widetilde{V}_{-1,-2,3,3}^{(4)} b_{2}^{*} b_{-3}^{* 2}
\end{aligned}
$$

$$
\begin{aligned}
a_{2}= & b_{2}+6 \widetilde{V}_{1,1,-2,2}^{(1)}\left(b_{-1} b_{-1}^{*} b_{-2}^{*}+b_{1} b_{1}^{*} b_{-2}^{*}\right)+6 \widetilde{V}_{2,2,-3,3}^{(1)}\left(b_{2} b_{3} b_{-3}+b_{2} b_{3}^{*} b_{-3}^{*}\right) \\
& +6 \widetilde{V}_{2,2,-1,1}^{(1)}\left(b_{2} b_{1} b_{-1}+b_{2} b_{1}^{*} b_{-1}^{*}\right)+6 \widetilde{V}_{3,3,-2,2}^{(1)}\left(b_{-3} b_{-3}^{*} b_{-2}^{*}+b_{3} b_{3}^{*} b_{-2}^{*}\right) \\
& +3 \widetilde{V}_{2,-1,3,3}^{(1)} b_{-1} b_{3}^{2}+3 \widetilde{V}_{1,-2,3,3}^{(1)} b_{-1} b_{-3}^{* 2}+3 \widetilde{V}_{3,-3,1,2}^{(1)} b_{1}^{*} b_{3} b_{-3}^{*} \\
& +2 \widetilde{V}_{-2,2,-1,1}^{(2)} b_{-2}^{*} b_{-1} b_{1}+2 \widetilde{V}_{-2,2,-3,3}^{(2)} b_{-2}^{*} b_{-3} b_{3}+2 \widetilde{V}_{3,-1,-3,2}^{(2)} b_{3} b_{-3}^{*} b_{-1} \\
& +3 \widetilde{V}_{2,2,-2,-2}^{(4)} b_{-2}^{* 2} b_{2}^{*}+\widetilde{V}_{-1,1,-2,2}^{(4)} b_{-1}^{*} b_{1}^{*} b_{-2}^{*}+\widetilde{V}_{-2,2,-3,3}^{(4)} b_{-2}^{*} b_{-3}^{*} b_{3}^{*} \\
& +3 \widetilde{V}_{1,2,-3,-3}^{(4)} b_{1}^{*} b_{-3}^{* 2} \\
a_{3} & b_{3}+ \\
+ & 6 \widetilde{V}_{1,1,-3,3}^{(1)}\left(b_{-1} b_{-1}^{*} b_{-3}^{*}+b_{1} b_{1}^{*} b_{-3}^{*}\right)+6 \widetilde{V}_{2,2,-3,3}^{(1)}\left(b_{-2} b_{-2}^{*} b_{-3}^{*}+b_{2} b_{2}^{*} b_{-3}^{*}\right) \\
+ & 6 \widetilde{V}_{3,3,-1,1}^{(1)}\left(b_{3} b_{1} b_{-1}+b_{3} b_{1}^{*} b_{-1}^{*}\right)+6 \widetilde{V}_{3,3,-2,2}^{(1)}\left(b_{3} b_{2} b_{-2}+b_{3} b_{2}^{*} b_{-2}^{*}\right) \\
+ & 6 \widetilde{V}_{2,-1,3,3}^{(1)} b_{2} b_{-1}^{*} b_{3}^{*}+6 \widetilde{V}_{1,-2,3,3}^{(1)} b_{1} b_{-2}^{*} b_{3}^{*}+3 \widetilde{V}_{3,-3,1,2}^{(1)}\left(b_{-3} b_{2} b_{1}+b_{-3} b_{-1}^{*} b_{-2}^{*}\right) \\
+ & 2 \widetilde{V}_{-3,3,-1,1}^{(2)} b_{-3}^{*} b_{-1} b_{1}+2 \widetilde{V}_{-3,3,-2,2}^{(2)} b_{-3}^{*} b_{-2} b_{2}+2 \widetilde{V}_{3,-1,-3,2}^{(2)}\left(b_{-3} b_{-2}^{*} b_{1}+b_{-3} b_{2} b_{-1}^{*}\right) \\
+ & 3 \widetilde{V}_{3,3,-3,-3}^{(4)} b_{-3}^{* 2} b_{3}^{*}+\widetilde{V}_{-1,1,-3,3}^{(4)} b_{-1}^{*} b_{1}^{*} b_{-3}^{*}+\widetilde{V}_{-2,2,-3,3}^{(4)} b_{-2}^{*} b_{2}^{*} b_{-3}^{*} \\
+ & 6 \widetilde{V}_{-1,-2,3,3}^{(4)} b_{-1}^{*} b_{-2}^{*} b_{3}^{*} .
\end{aligned}
$$

Similarly $a_{-j}$ is given by replacing $k_{j}$ by $-k_{j}$ in Eqs.(44), (45), and (46).

The coefficients $\widetilde{V}_{i, j, l, m}^{(1)}, \widetilde{V}_{i, j, l, m}^{(2)}$ and $\tilde{V}_{i, j, l, m}^{(4)}$ are given by :

$$
\begin{aligned}
& \tilde{V}_{i, j, l, m}^{(1)}=\frac{V_{i, j, l, m}^{(1)}}{\omega_{i}-\omega_{j}-\omega_{l}-\omega_{m}}, \\
& \widetilde{V}_{i, j, l, m}^{(2)}=\frac{V_{i, j, l, m}^{(2)}}{\omega_{i}+\omega_{j}-\omega_{l}-\omega_{m}} \text { for } \omega_{i}+\omega_{j} \neq \omega_{l}+\omega_{m}, \\
& \tilde{V}_{i, j, l, m}^{(4)}=\frac{V_{i, j, l, m}^{(4)}}{\omega_{i}+\omega_{j}+\omega_{l}+\omega_{m}} .
\end{aligned}
$$


Remark 1: We notice that when the modes of one resonance are equal to zero, e.g, $b_{-j}=0$ for $j=1,2,3$ then we have $a_{j}=b_{j}$ for the second resonance $(j=1,2,3)$.

Remark 2: When two modes of each resonance are equal to zero e.g $b_{j}=b_{-j}=0$ for $j=1,3$ then $a_{2}=b_{2}+2 \widetilde{V}_{2,2,-2,-2}^{(4)} b_{-2}^{* 2} b_{2}^{*}$, where $\widetilde{V}_{2,2,-2,-2}^{(4)}=-0.08188$.

The transformation which maps $\left\{a_{j}\right\}$ into $\left\{b_{j}\right\}$ is canonical i.e. it satisfies

$$
\sum_{j} i d b_{j} \wedge d b_{j}^{*}=\sum_{j} i d a_{j} \wedge d a_{j}^{*}
$$

This transformation maps Hamiltonian (43) into Hamiltonian (8) with the following values of the coefficients $U_{i}, \mathrm{~W}$ and $V_{i j}$.

\begin{tabular}{|c|c|c|c|}
\hline$U_{1}$ & $U_{2}$ & $U_{3}$ & $\mathrm{~W}$ \\
\hline 0.0063 & 4.61645 & 0.40528 & 0.52648 \\
\hline
\end{tabular}

\begin{tabular}{|c|c|c|c|c|c|}
\hline$V_{21}$ & $V_{-21}$ & $V_{31}$ & $V_{-31}$ & $V_{23}$ & $V_{-23}$ \\
\hline 0.07599 & 0.53193 & 0.02533 & 0.17312 & 5.16738 & 2.12774 \\
\hline
\end{tabular}

[1] T.B. Benjamin and J.E. Feir, "The disintegration of wave trains in deep water, Part1. Theory", J. Fluid Mech. 27, 417, (1967).

[2] M.J. Lighthill, "Some special cases treated by Witham theory", Proc. R. Soc. London, Ser. A 299, 28, (1967).

[3] V.E. Zakharov, "Stability of periodic waves of finite amplitude on the surface of a deep fluid", J. Appl. Mech. Tech. Phys. (U.S.S.R.), 51. 269, (1968).

[4] B.M Lake, HC. Yuen, H. Rungaldier and W.E. Ferguson, "Nonlinear deep-water waves: theory and experiment. Part 2. Evolution of a continuous wave train", J. Fluid Mech. 83, 49, (1977).

[5] E. Fermi, J. Pasta and S. Ulam, "Studies of nonlinear problems", "Collected Papers of Enrico Fermi", University of Chicago Press, Chicago, Illinois, 2, 978, (1955).

[6] W. Craig, "Birkhoff normal forms for water waves", Contemp. Math. 200, 57,(1996).

[7] W. Craig, M.D. Groves, "Hamiltonian long-wave approximations to the water-wave problem", Wave Motion 19, 367, (1994).

[8] F. Dias and C. Kharif, "Nonlinear gravity and capillary-gravity waves", Ann.Rev. Fluid Mech. 31, 301, (1999). 
[9] L. Shemer and M. Stiassnie, "Initial instability and long-time evolution of Stokes waves in the ocean surface: Wave breaking, turbulent Mixing and Radio Probing", edited by Y.Toba and H.Mitsuyasu. (Reidel Publishing Co., Dordrecht, 1985).

[10] M. Stiasnie and L. Shemer, "Energy computations for evolution of class I and II instabilities of Stokes waves", J. Fluid.Mech. 174, 299, (1987).

[11] V.I. Shrira, S.I. Badulin and C. Kharif, "A model of water wave horse-shoe patterns", J. Fluid Mech. 318, 375, (1996).

[12] S.I. Badulin, V.I. Shrira, C. Kharif and M. Ioualalen, "On two approaches to the problem of instability of short-crested water waves", J. Fluid Mech. 303, 272, (1995).

[13] T. Benzekri, R. Lima and M. Vittot, "Non-permanent form solutions in the Hamiltonian formulation of surface water waves", Eur. J. Mech. B-Fluids 19, 379, (2000).

[14] E.A. Caponi, P.G. Saffman and H.C. Yuen, "Instability and confined chaos in nonlinear dispersive wave system", Phys. Fluids 25, 2159, (1982).

[15] J.A. Zufiria, "Oscillatory spatial periodic weakly nonlinear gravity waves on deep water", J. Fluid Mech. 191, 341, (1988).

[16] W. Craig, M.D. Groves, "Normal forms for wave motion in fluid interfaces", Wave Motion 31, $21(2000)$.

[17] F. Dias, T.J. Bridges, "Geometric aspects of spatially periodic interfacial waves", Studies Appl. Math. 93, 93, (1994).

[18] V.P. Krasitskii, "On reduced equations in the Hamiltonian theory of weakly nonlinear surface waves", J. Fluid Mech. 272, 1, (1994).

[19] A.I. Dyachenko and V.E. Zakharov, "Is free surface Hydrodynamics an integrable system?", Phys. Lett. A 190, 144, (1994).

[20] W. Craig and P. Worfolk, "An integrable normal form for water waves in infinite depth", Physica D 84, 513, (1995). 
FIG. 1: Representation of the phase space $\mathbb{E}_{+}$and the three families of fixed points found on $\left(N_{1}, \theta_{1}\right)$ plane. The location of the elliptic points $e\left(\mathbb{I}_{+}\right), e_{1}\left(\mathbb{I}_{+}\right)$is represented by a dashed line. The location of the hyperbolic points $h_{1}\left(\mathbb{I}_{+}\right)$is represented by a dotted line. The bifurcation creating the pair of elliptic/hyperbolic $e_{1}\left(\mathbb{I}_{+}\right) / h_{1}\left(\mathbb{I}_{+}\right)$point is indicated by a dot.

FIG. 2: Elevation of the surface $\eta(x, t)$ corresponding to $P\left(\mathbb{I}_{+}=0\right)$ for Hamiltonian (12) with one resonance.

FIG. 3: Phase space trajectories of Hamiltonian (12) with one resonance for different values of $\mathbb{I}_{+}:(a) \mathbb{I}_{+}=0.1,(b) \mathbb{I}_{+}=0.12,(c) \mathbb{I}_{+}=0.5,(d) \mathbb{I}_{+}=0.505,(e) \mathbb{I}_{+}=0.6,(f) \mathbb{I}_{+}=1.7 .$, The grey region is the accessible region of phase space: which is the annulus $\max \left(0, \mathbb{K}_{+}\right) \leq N_{1} \leq \mathbb{I}_{+} / 2$. The bold curve $\mathcal{T}_{1}$ represents the trajectory which passes by $N_{1}=0$

FIG. 4: Elevation of the surface $\eta(x, t)$ for Hamiltonian (12) with one resonance for $\mathbb{J}_{+}=1$, $\mathbb{I}_{+}=\mathbb{J}_{+} / 2$, and for the fixed point $e\left(\mathbb{J}_{+} / 2\right)$.

FIG. 5: Temporal evolution of modes $N_{i}$ for $i=1,2,3$, for Hamiltonian (18) with one resonance, $\mathbb{I}_{+}=1.2, \mathbb{J}_{+}=1$ and an initial condition $\left(N_{1}, \theta_{1}\right)=\left(\mathbb{I}_{+} / 2, \pi\right)$.

FIG. 6: Temporal evolution of modes $N_{i}$ for $i=1,2,3$, for Hamiltonian (18) with one resonance , $\mathbb{J}_{+}=1, \mathbb{I}_{+}$in the neighborhood of $\mathbb{I}_{+}=\mathbb{J}_{+} / 2$, and an initial condition $\left(N_{1}, \theta_{1}\right)=\left(\mathbb{J}_{+} / 8,0\right)$.

FIG. 7: Elevation of the surface $\eta(x, t)$ Hamiltonian (18) with one resonance for $\mathbb{J}_{+}=1$, in the neighborhood of $\mathbb{I}_{+}=\mathbb{J}_{+} / 2$, and and an initial condition $\left(N_{1}, \theta_{1}\right)=\left(\mathbb{J}_{+} / 8,0\right)$

FIG. 8: Temporal evolution of modes $N_{i}$ for $i=1,2,3$, for Hamiltonian (18) with one resonance , $\mathbb{J}_{+}=1, \mathbb{I}_{+}=\mathbb{J}_{+} / 2$, and an initial condition on the separatrix $\left(N_{1}, \theta_{1}\right)=\left(\mathbb{J}_{+} / 4,2.8\right)$.

FIG. 9: Elevation of the surface $\eta(x, t)$ for Hamiltonian (18) with one resonance for $\mathbb{J}_{+}=1$, $\mathbb{I}_{+}=\mathbb{J}_{+} / 2$, and for the fixed point $\left(N_{1}, \theta_{1}\right)=\left(\mathbb{J}_{+} / 4,2.8\right)$ 
FIG. 10: Plots of $\eta(x, t)$ for Hamiltonian (8) with two resonances and for different values of $\alpha$ : $(a)$ standing wave $\alpha=1,(b)$ stationary wave $\alpha$ indefinite $c=0,(c)$ traveling wave $\alpha=-1,(d)$ time periodic wave $\alpha \in \mathbb{Q}\left(-\mathbb{J}_{+}+2 \mathbb{J}_{-}=J_{0}\right),(e)$ time quasiperiodic wave $\alpha=\sqrt{0.4}$.

FIG. 11: Poincaré sections for Hamiltonian (38) with two resonances for $H=2, \mathbb{J}_{+}=\mathbb{J}_{-}=1$ and for different values of $\mathbb{I}_{+}:(a) \mathbb{I}_{+}=\mathbb{I}_{-}=0.3,(b) \mathbb{I}_{+}=\mathbb{I}_{-}=0.4,(c) \mathbb{I}_{+}=\mathbb{I}_{-}=0.5,(d) \mathbb{I}_{+}=\mathbb{I}_{-}=0.8$. The grey region is the accessible region where $\max \left(0, \mathbb{K}_{+}\right) \leq \mathrm{N}_{1} \leq \mathbb{I}_{+} / 2$.

FIG. 12: Time evolution of $N_{j}$ for $j=-1,-2,-3,1,2,3$, for Hamiltonian (38) with two resonances and for $\mathbb{I}_{+}=\mathbb{I}_{-}=0.5$ and for an initial condition in the chaotic region $\left(N_{1}, \theta_{1}\right)=(0.152,-3.07)$.

FIG. 13: Shape of the wave $\eta(x, t)$ for $\mathbb{I}_{+}=\mathbb{I}_{-}=0.5$ for Hamiltonian (38) with two resonances and for an initial condition in the chaotic region $\left(N_{1}, \theta_{1}\right)=(0.152,-3.07)$. 